\title{
Chemogenetic silencing of hippocampal neurons suppresses epileptic neural circuits
}

\author{
Qi-Gang Zhou, ${ }^{1,2}$ Ashley D. Nemes, ${ }^{3}$ Daehoon Lee, ${ }^{1}$ Eun Jeoung Ro, ${ }^{1}$ Jing Zhang, ${ }^{2}$ Amy S. Nowacki, ${ }^{4}$ Susan M. Dymecki, ${ }^{5}$ \\ Imad M. Najm, ${ }^{3}$ and Hoonkyo Suh ${ }^{1}$ \\ 'Department of Neurosciences, Cleveland Clinic, Cleveland, Ohio, USA. '2Department of Clinical Pharmacology, Pharmacy College, Nanjing Medical University, Nanjing, China. ${ }^{3}$ Epilepsy Center, \\ Neurological Institute, and ${ }^{4}$ Department of Quantitative Health Sciences, Cleveland Clinic, Cleveland, Ohio, USA. ${ }^{5}$ Department of Cenetics, Harvard Medical School, Boston, Massachusetts, USA.
}

\begin{abstract}
We investigated how pathological changes in newborn hippocampal dentate granule cells (DCCs) lead to epilepsy. Using a rabies virus-mediated retrograde tracing system and a designer receptors exclusively activated by designer drugs (DREADD) chemogenetic method, we demonstrated that newborn hippocampal DCCs are required for the formation of epileptic neural circuits and the induction of spontaneous recurrent seizures (SRS). A rabies virus-mediated mapping study revealed that aberrant circuit integration of hippocampal newborn DCCs formed excessive de novo excitatory connections as well as recurrent excitatory loops, allowing the hippocampus to produce, amplify, and propagate excessive recurrent excitatory signals. In epileptic mice, DREADD-mediated-specific suppression of hippocampal newborn DCCs dramatically reduced epileptic spikes and SRS in an inducible and reversible manner. Conversely, specific activation of hippocampal newborn DCCs increased both epileptic spikes and SRS. Our study reveals an essential role for hippocampal newborn DCCs in the formation and function of epileptic neural circuits, providing critical insights into DCCs as a potential therapeutic target for treating epilepsy.
\end{abstract}

\section{Introduction}

Epilepsy is the third most common neurological disorder in the United States, and more than 2 million people (an incidence of approximately 1 in 100) suffer from this condition. Unfortunately, $30 \%-40 \%$ of epilepsy patients become medically refractory and fail to respond to current medical treatments $(1,2)$. Temporal lobe epilepsy (TLE) falls into the category of medically intractable epilepsy, as its etiology is unknown and therapeutic options are limited $(3,4)$. TLE often initiates at a focal structure, such as the hippocampus, and eventually spreads throughout the brain after a seizure-free latent period (5). This observation has led to the hypothesis that pathological changes in the hippocampus during the latent period are a critical step in the development of the spontaneous recurrent seizures (SRS) that are characteristic of TLE; however, the nature of these pathological changes responsible for the onset of SRS is not clearly understood.

Recent studies have supported an emerging view that adultborn hippocampal dentate granule cells (DGCs) are directly involved in epileptogenesis (6-8). DGCs are excitatory neurons that are continuously generated and integrated into hippocampal neural circuits, and this neurogenesis is critical for cognition and emotion (9). Conditional deletion of Pten in hippocampal newborn DGCs led to the development of spontaneous epilepsy that resembled TLE (7). In a pilocarpine-induced status epilepticus (SE) rodent model that closely mimics TLE, genetic ablation of hippocampal newborn DGCs prior to or immediately after SE induction

Conflict of interest: The authors have declared that no conflict of interest exists. License: Copyright 2019, American Society for Clinical Investigation.

Submitted: June 15, 2017; Accepted: October 30, 2018.

Reference information: J Clin Invest. 2019;129(1):310-323.

https://doi.org/10.1172/JCI95731 effectively reduced the development of SRS $(6,8)$, indicating that pathological changes in a small population of hippocampal DGCs are sufficient to induce epilepsy. Morphological analysis has also suggested that abnormal neuronal development of newborn DGCs may play a critical role in epileptogenesis. Indeed, studies of human TLE patients and TLE rodent models showed that adult-born DGCs developed abnormally, displaying mossy fiber sprouting (10-14), abnormal hilar basal dendrite formation $(15,16)$, and ectopic dispersion and migration to the hilus or CA3 $(17,18)$. DGCs that have ectopically formed basal dendrites or that have abnormally migrated to the hilus or CA3 receive excessive excitatory input from mossy fibers or CA3 pyramidal neurons. Moreover, sprouting of mossy fibers of DGCs forms additional excitatory connections onto neighboring DGCs. These structural and migratory abnormalities of DGCs have been proposed to be a key factor in the formation of proepileptic neural circuits. Interestingly, this vulnerability to structural deficits is determined by the age of DGCs relative to an epileptic insult: while DGCs that were already mature at the time of SE did not show major structural abnormalities, immature DGCs or DGCs that were born immediately after SE insult were vulnerable to epileptogenic pathological changes (19-21).

Although these results have raised the interesting possibility that abnormal and pathological changes in hippocampal newborn DGCs contribute to proepileptic neural circuits, the lack of suitable methods to precisely map and manipulate altered neural circuits has hampered investigation into the direct role of hippocampal newborn DGCs in epilepsy. In this study, using recently developed methods to trace and manipulate neural circuits, we demonstrated that aberrant recruitment of hippocampal newborn DGCs formed de novo excessive recurrent excitatory networks that rendered synchronous discharges and induced spontaneous epilepsy. Anatomically, using a rabies virus-mediated retrograde 
A

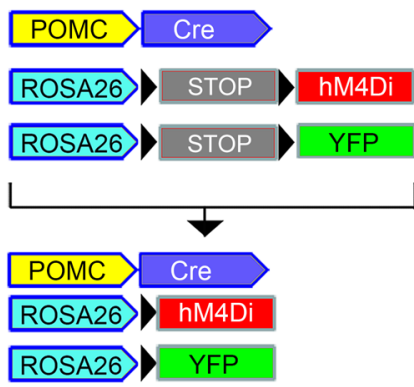

POMC-Cre;hM4Di ${ }^{\mathrm{fl} /+} ; \mathrm{YFP}^{\mathrm{fl} /+}$ or POMC-Cre;hM4Dit/+
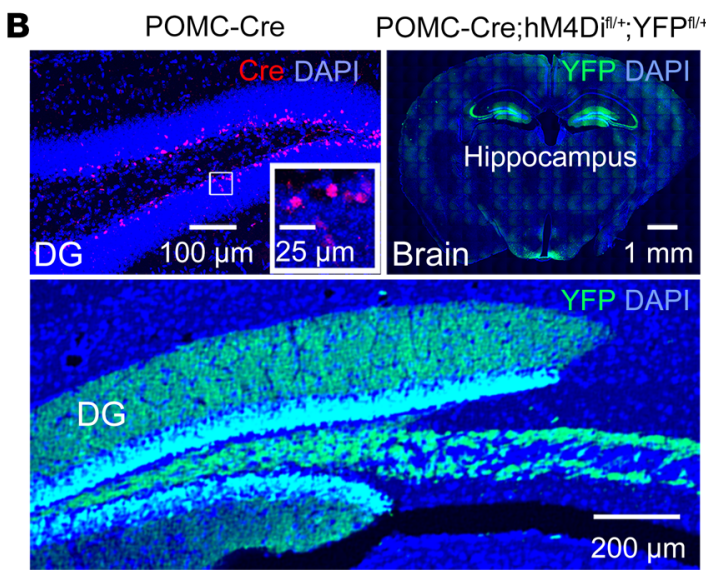

C
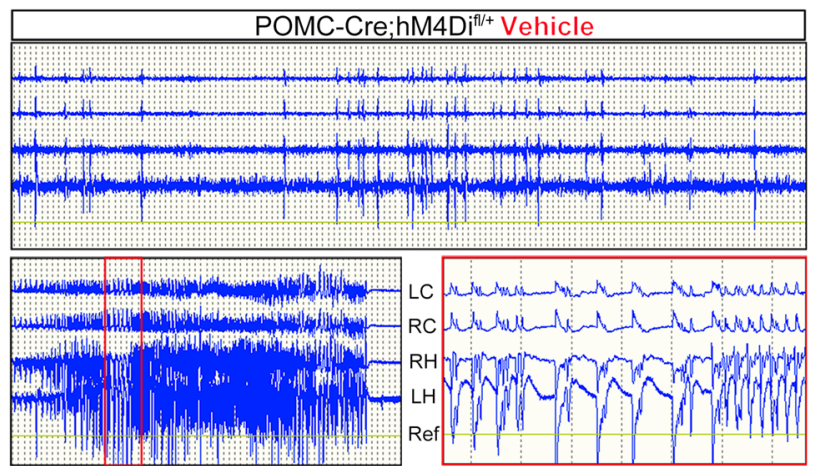

D

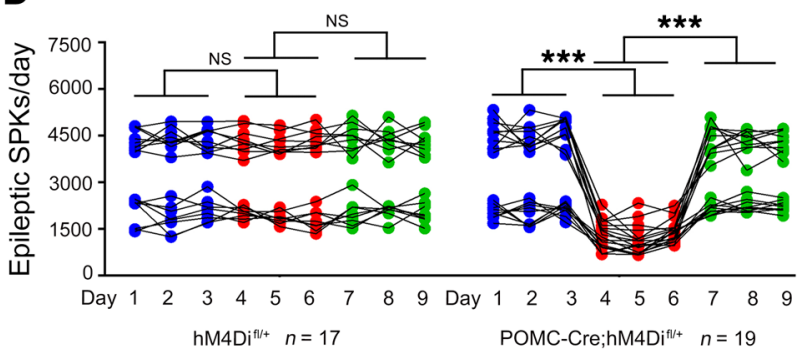

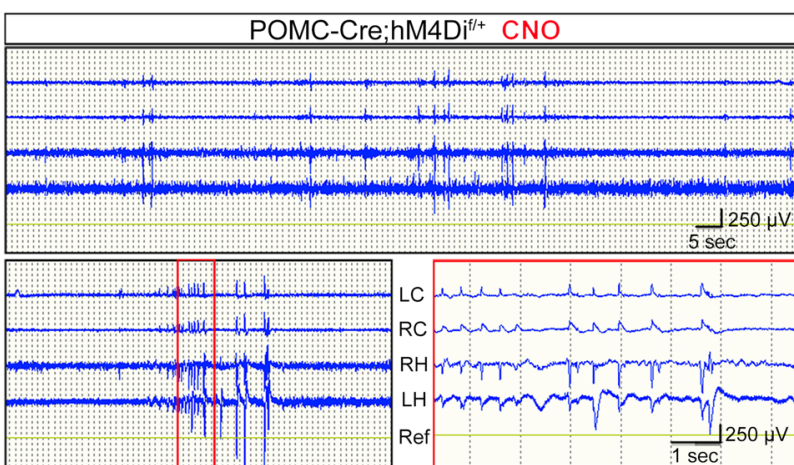

E

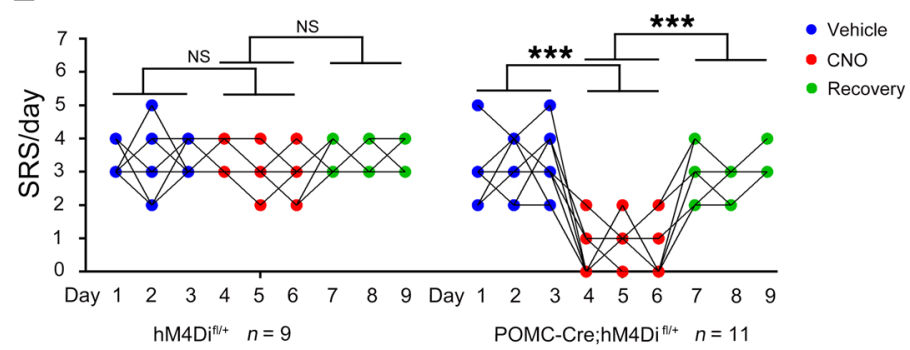

Figure 1. Inhibition of hippocampal DGCs suppresses SRS. (A) hM4Di and YFP are expressed in hippocampal DGCs in POMC-Cre; hM4Difl/+;YFPfl/+ mice. (B) CRE is expressed in the DC, and YFP, which is an indicator of hM4Di, is expressed in all DGCs in POMC-Cre; hM4Di $\mathrm{i}^{\mathrm{fl} /+} ; \mathrm{YFP} \mathrm{fl}^{\mathrm{f} /+}$ mice. (C) Representative EEG traces in the absence and presence of CNO in POMC-Cre; hM4Di ${ }^{\mathrm{fl} /+}$ mice during pilocarpine-induced epilepsy. (D and E) On days 1-3, vehicle treatment did not significantly change epileptic spikes or SRS in either control or POMC-Cre;hM4Difl/+ mice. On days 4-6, CNO-mediated suppression of hippocampal DCCs significantly reduced epileptic spikes (SPKs) $(n=19)$ as well as SRS ( $n=11)$ in POMC-Cre;hM4Difl/+ mice in an inducible and reversible manner, as determined by 2-way RM ANOVA with a Bonferroni's multiple comparison post test. On days 7-9, epileptic spikes and SRS returned to basal levels in POMC-Cre; hM4Difl/+, showing CNO-dependent transient and reversible suppression of DGC activity. Note that epilepsy spikes and SRS were quantified during the 24 hours after vehicle (days 1-3, blue circles), CNO (days 4-6, red circles), and recovery without treatment (days 7-9, green circles). Two-way RM ANOVA with Bonferroni's multiple comparison tests were used for $\mathbf{D}$ and $\mathbf{E}$. Asterisks indicate that CNO treatment resulted in a significant reduction in epileptic spikes and SRS compared with vehicle treatment, which returned to basal levels during the recovery period. ${ }^{* *} P<0.001$.

tracing method (22), we precisely mapped aberrant connectivity of hippocampal newborn DGCs with their afferent (input) neurons in epileptic mice. This approach revealed that hippocampal newborn DGCs increased connections with excitatory afferent neurons, substantially increasing the connectivity ratio of excitatory-to-inhibitory inputs to DGCs. Increased interconnections between DGCs (within the dentate gyrus) as well as reciprocal connections between DGCs and CA3 pyramidal neurons (between the dentate gyrus and CA3) formed recurrent excitatory loops that produce and amplify excessive excitatory signals in the dentate gyrus and allow them to propagate through the hippocampus. In addition, we showed that the relative age of DGCs with respect to the initial SE is a critical factor that determines whether newborn DGCs are vulnerable to recruitment into proepileptic neural circuits. To understand the functional significance of this aberrant integration of hippocampal newborn DGCs in seizure expression during epilepsy, we used a designer receptors exclusively activated by designer drugs (DREADD) method (23-25) that allowed us to manipulate the neuronal activity of hippocampal DGCs in an inducible and reversible manner. Specific suppression of hippo- 
A I RV-Syn-GTRgp i RbV-EnvA- $\triangle$ Rgp-MCh
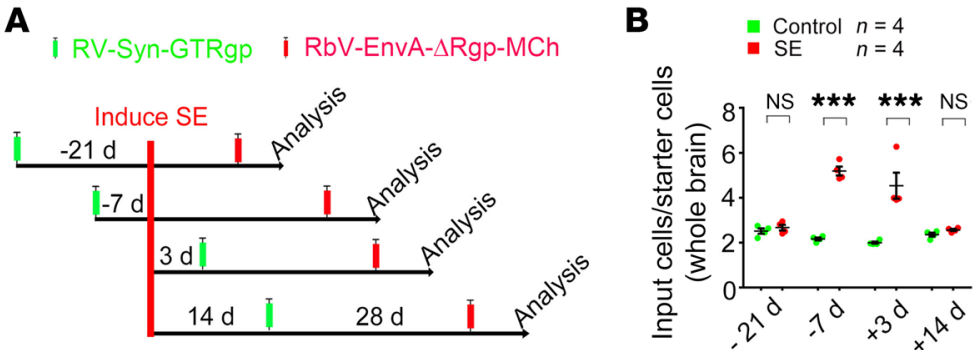

C

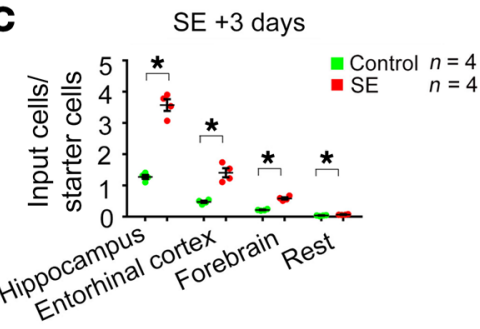

E

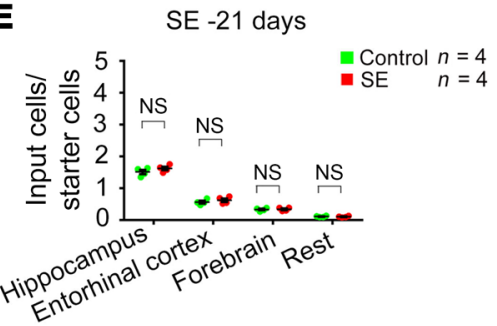

G
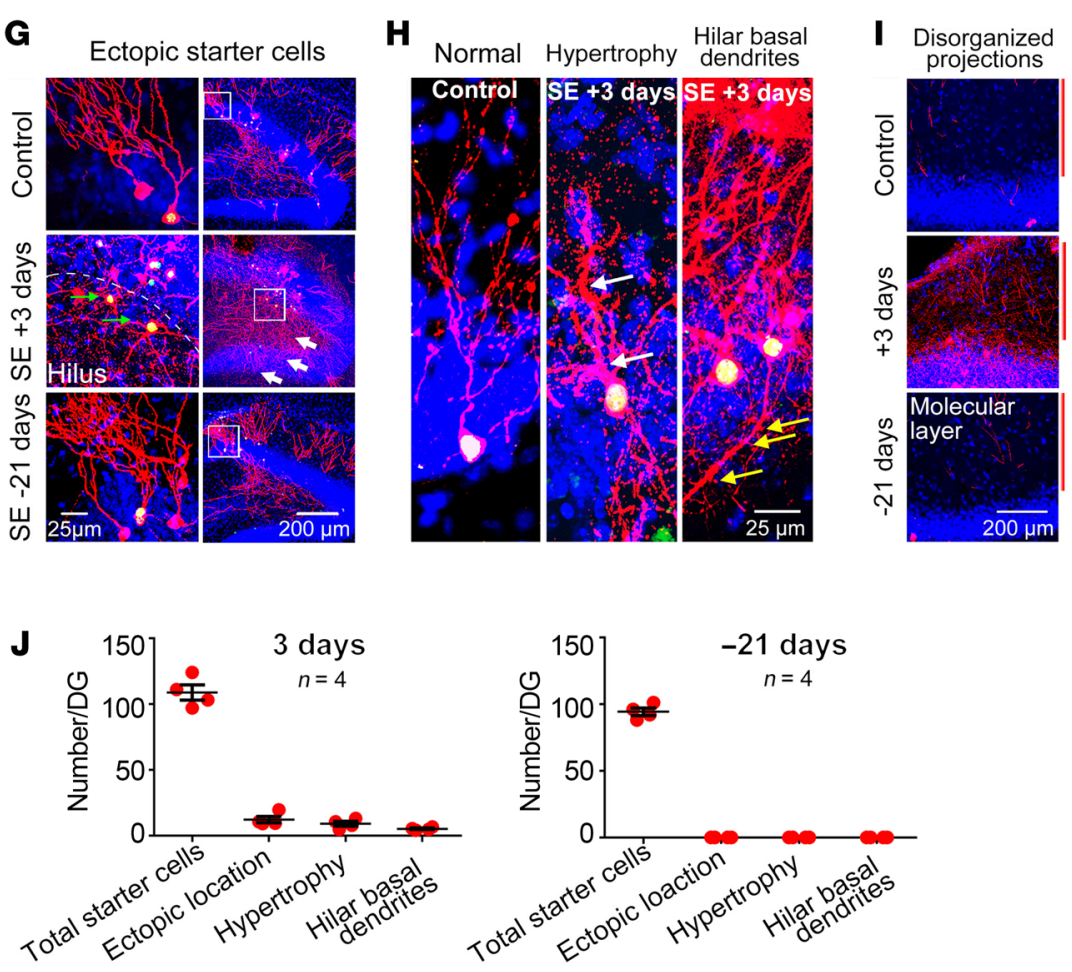

Figure 2. Aberrant integration of DGCs into proepileptic neural circuits depends upon the age of DGCs. (A) Experimental schematic showing mapping of neu ronal connectivity of DCCs born before and after SE induction. (B) The connectivity ratio of input neurons to starter cells shows the critical time window during which newborn DCCs of different ages are recruited to proepileptic neural circuits ( $n=4$, each). (C-F) Connectivity ratios of DCCs born 3 and 14 days after SE and 7 and 21 days before SE are shown. DGCs born between 7 days before and 3 days after SE induction show aberrant connectivity, whereas the connectivity of DGCs born 21 days before or 14 days after SE induction is not significantly different $(n=4$, each). (G-J) DGCs born 3 days after SE, but not those born 2 days prior to $\mathrm{SE}$, are ectopically located, are hypertrophic, and develop hilar basal dendrites ( $n=4$, each) Arrows ( $\mathbf{G}$ and $\mathbf{H}$ ) indicate disorganized projections. Disorganized axonal projections were evident when the input of DCCs born 3 days after, but not 21 days before, SE induction was examined. Red lines on the right side of each image (I) indicate the location of projections. ${ }^{*} P<0.05 ;{ }^{* *} P<0.001,2$-way ANOVA with Bonferroni's multiple comparison tests (B) and Mann-Whitney nonparametric $U$ tests (C-F).

essential role of hippocampal newborn DGCs in proepileptic neural circuits and the critical time window during which newborn DGCs are pathologically integrated into proepileptic neural circuits, which provides a potential time point for therapeutic intervention.

\section{Results}

Hippocampal DGCs play an essential role in epileptic spike and SRS induction. To determine whether hippocampal DGCs are directly involved in the occurrence of epileptic seizures, a DREADD method was used to inactivate the neuronal activity of both mature and newborn DGCs (23). DREADDs are engineered G protein-coupled human muscarinic acetylcholine receptors that do not react to any endogenous ligands. However, upon the administration of clozapine $\mathrm{N}$-oxide (CNO), which is a pharmacologically inert drug, hM3Dq or hM4Di DREADD receptors activated or suppressed neuronal activity, respectively. In this study, genetically engineered mice $\left(\mathrm{hM} 4 \mathrm{Di}^{\mathrm{fl} /+}\right)$ were used to express hM4Di receptors in a Credependent manner (24).

To determine the specific expression of hM4Di receptors in DGCs and to examine the efficacy of CNO-mediated suppression of DGC neuronal activity, we generated triple-transgenic mice by crossing POMC-Cre, ROSA26-hMcampal newborn DGCs was sufficient to decrease the occurrence of SRS, while specific activation of hippocampal newborn DGCs dramatically increased SRS, demonstrating that hippocampal newborn DGCs are both necessary and sufficient for the occurrence of SRS in epileptic mice. Collectively, our study reveals an
4Di, and ROSA26-YFP reporter mice (POMC-Cre;hM4Difl/; $\mathrm{YFP}^{\mathrm{fl} /+}$; Figure 1A). In this triple-transgenic mouse, the proopiomelanocortin (POMC) promoter transiently expresses a CRE recombinase in immature DGCs $(26,27)$; the recombinase deletes a stop codon flanked by 2 loxP sites, leading to the 
expression of inhibitory hM4Di receptors and the YFP reporter gene in DGCs. YFP reporter expression confirmed the specific expression of hM4Di receptors in both mature and immature DGCs (Figure 1B). Using c-FOS as a surrogate marker for neuronal activity, the dose and kinetics of $\mathrm{CNO}$-mediated inhibition of DGC neuronal activity were examined (Supplemental Figure 1, A-E; supplemental material available online with this article; https://doi.org/10.1172/JCI95731DS1). A CNO dose higher than $10 \mathrm{mg} / \mathrm{kg}$ was sufficient to reduce the number of c-FOS ${ }^{+}$cells to $25 \%$ or lower, which lasted for at least 2 to 4 hours after CNO administration (Supplemental Figure 1, A and B). This CNOmediated inhibition of neuronal activity was specific to DGCs located in the dentate gyrus, as neurons in other brain areas, such as CA3, CA1, and the cortex, were unaffected (Supplemental Figure 2). These results collectively demonstrate that the neuronal activity of DGCs can be tightly regulated in an inducible manner by using the DREADD method.

Using a pilocarpine-mediated SE mouse model that efficiently and faithfully exhibits spontaneous epilepsy (Supplemental Figure 3), we investigated the direct involvement of DGCs in seizure generation during epilepsy. At 10 weeks after the initial induction of SE, when both hM4Di $\mathrm{i}^{\mathrm{fl}+}$ and POMC-Cre;hM4 $4 \mathrm{Di}^{\mathrm{il} /+}$ mice stably developed spontaneous epilepsy (Figure 1C and Supplemental Figure 3, E-G), the effects of reduced DGC activity on seizure expression were examined by injecting CNO. On days 1-3, treatment with vehicle did not significantly change epileptic spikes or SRS in either hM4 $\mathrm{Di}^{\mathrm{fl} /+}$ or POMC-Cre;hM4Difl/+ mice (Figure 1, $\mathrm{C}-\mathrm{E})$. However, administration of $\mathrm{CNO}$ on days 4-6 significantly reduced the frequency of epileptic spikes and the occurrence of SRS, specifically in POMC-Cre;hM4Difl//+ mice, but not in hM4Difl/t+ control mice (Figure 1, C-E). Epileptic spikes and SRS returned to basal levels when measured in the absence of $\mathrm{CNO}$ on days 7-9 (Figure 1, D and E). In addition, using a separate cohort of mice, we repeatedly injected CNO into the same mice over a 7-day interval. CNO administration reproducibly decreased both epileptic spikes and SRS exclusively in POMC-CRE;hM4Difl/+ mice, but not in control mice (Supplemental Figure 4, A-D). These results clearly show that the DREADD method produced inducible and reversible effects and that the suppression of epileptic phenotypes was dependent upon the expression of inhibitory hM4Di receptors in DGCs and the availability of CNO.

To rule out the possibility that POMC-expressing neurons located outside the hippocampus may be involved in the suppression of epilepsy, CNO or vehicle was directly administered into the hippocampus of $\mathrm{hM} 4 \mathrm{Di}^{\mathrm{il} /+}$ and POMC-Cre;hM4 $\mathrm{Di}^{\mathrm{fl} /+}$ mice that developed spontaneous epilepsy following SE (Supplemental Figure 5). One day after CNO infusion into the hippocampus of POMC-Cre;hM$4 \mathrm{Di}^{\mathrm{il} /+}$ mice, the frequency of epileptic spikes and SRS, as well as the number of c-FOS ${ }^{+}$DGCs, were significantly reduced (Supplemental Figure 5, C and F) compared with those in vehicle-treated mice. CNO administration into control mice $\left(\mathrm{hM} 4 \mathrm{Di}^{\mathrm{fl} /+}\right)$ did not alter epileptic spikes, SRS, or the number of c-FOS ${ }^{+}$DGCs. Seven days after the direct hippocampal administration of $\mathrm{CNO}$, both epileptic spikes and SRS returned to basal levels in epileptic POMC-Cre;hM$4 \mathrm{Di}^{\mathrm{fl} /+}$ mice (Supplemental Figure 5, C and G). These results collectively demonstrate the essential role of hippocampal DGCs in the expression of epileptic spikes and SRS during epilepsy.
Aberrant integration of newborn DGCs into hippocampal neural circuits. Previous studies have identified the age of DGCs relative to the onset of SE as a critical factor that determines whether newborn DGCs are subject to abnormal structural development $(20,21,28)$. To test the possibility that aberrant circuit integration is also dependent upon the age of hippocampal DGCs, we mapped neuronal connectivity of hippocampal DGCs with afferent input neurons using a rabies virus-mediated retrograde tracing method (22). We used an engineered rabies virus with 2 major modifications that secured its specific infection into newborn DGCs and monosynaptic spread to first-order afferent neurons. First, a rabies virus was engineered in which the gene encoding rabies virus glycoprotein (Rgp), which is essential for transsynaptic propagation in a retrograde manner, was replaced with an mCherry $(\mathrm{MCh})$ reporter gene (RbV- $\Delta \mathrm{Rgp}$ $\mathrm{MCh})(29,30)$. Second, the RbV- $\Delta \mathrm{Rgp}-\mathrm{MCh}$ virus was pseudotyped with an avian virus envelope protein (EnvA) (RbV-EnvA- $\Delta$ Rgp$\mathrm{MCh}$ ), which only recognizes an avian cell-specific receptor, TVA. Third, to ensure the specific infection of rabies into hippocampal newborn DGCs, we generated a retrovirus (RV) that expresses a histone-tagged, nuclear-localized GFP (hGFP), TVA receptor, and Rgp under the control of the neuronal-specific synapsin promoter (RV-Syn-GTRgp). The RV-Syn-GTRgp virus exclusively infects proliferating neural stem cells that differentiate into DGCs and express hGFP, TVA, and Rgp in an age-synchronized manner.

To determine the neuronal connectivity of hippocampal DGCs, the retrovirus RV-Syn-GTRgp was first injected into the dentate gyrus 21 or 7 days before, as well as 3 or 14 days after, the onset of pilocarpine-induced SE (Figure 2A). Twenty-eight days later, RbV-EnvA- $\triangle$ Rgp-MCh rabies virus was injected into the same locations where the RV had been previously injected, and the brains were then analyzed 1 week later. RbV-EnvA- $\Delta$ Rgp-MCh selectively infects newborn neurons that are labeled with RV-SynGTRgp by the specific interaction between EnvA present in the RbV and a TVA receptor expressed by the RV-Syn-GTRgp in newborn DGCs. This RbV is then complemented by Rgp (produced only in newborn DGCs infected by RV-Syn-GTRgp) and crosses synapses only once, in a retrograde manner, expressing the MCh reporter in afferent neurons that directly project to starter newborn DGCs. The starter DGCs were identified by coexpression of hGFP (from RV-Syn-GTRgp) and MCh (from RbV-EnvA- $\Delta$ Rgp$\mathrm{MCh}$ ), and input neurons were identified by the expression of MCh (from RbV-EnvA- $\Delta$ Rgp-MCh) $(22,31)$.

SE globally changed the connectivity ratio, which was defined as the number of input neurons per starter DGC, and such changes were dependent upon the relative age of DGCs with respect to the onset of SE (Figure 2B). New DGCs born 7 days before or 3 days after SE showed significant increases in connectivity ratio, while the connectivity ratios of DGCs born 21 days before or 14 days after SE were not altered (Figure 2B). Hippocampal newborn DGCs received diverse axonal innervation from input neurons originating in many different brain structures, and most of these input neurons conveyed excitatory signals. DGCs born 7 days before or 3 days after $\mathrm{SE}$ received increased inputs from neurons located in the entorhinal cortex (EC), forebrain, brain stem, and hippocampus (Figure 2, C and D); however, DGCs born 21 days before and 14 days after SE showed no significant changes in connectivity ratio with input neurons from any of these areas (Figure 2, E and F). 
A

RV-Syn-GTRgp RbV-EnvA- $\Delta$ Rgp-MCh

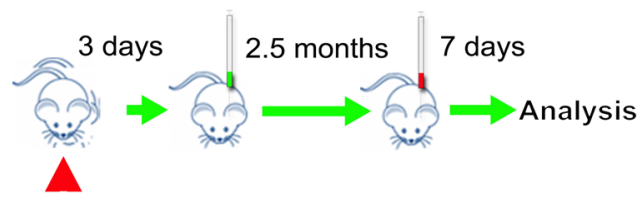

Induce SE

E Control $n=4$ SE 3 days $n=4$

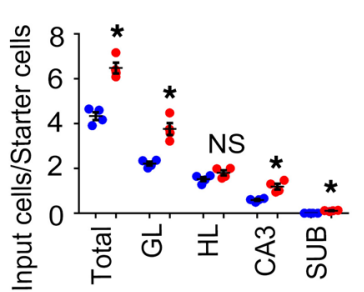

Intra-hippocampus
Entorhinal cortex

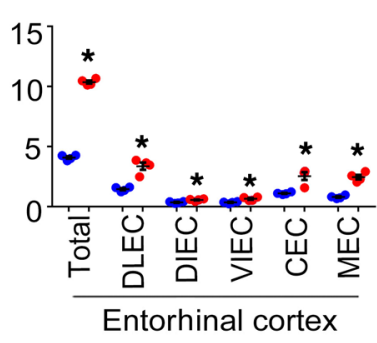

B

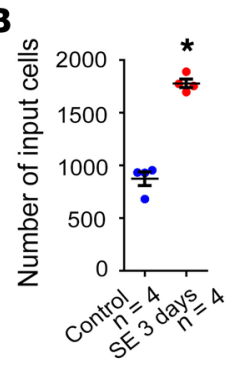

C

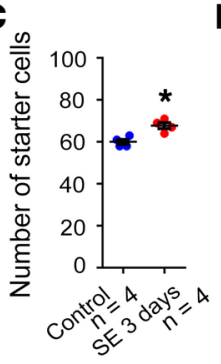

D

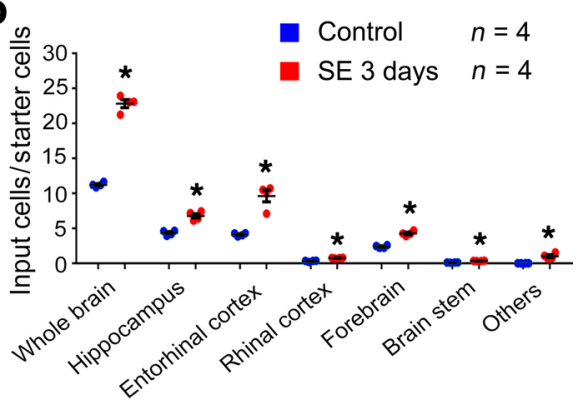

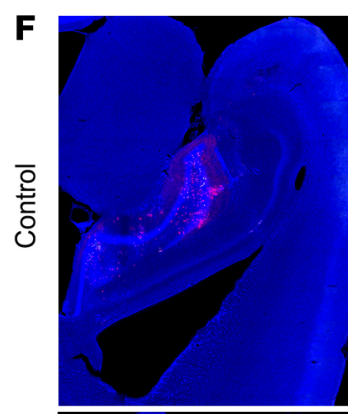
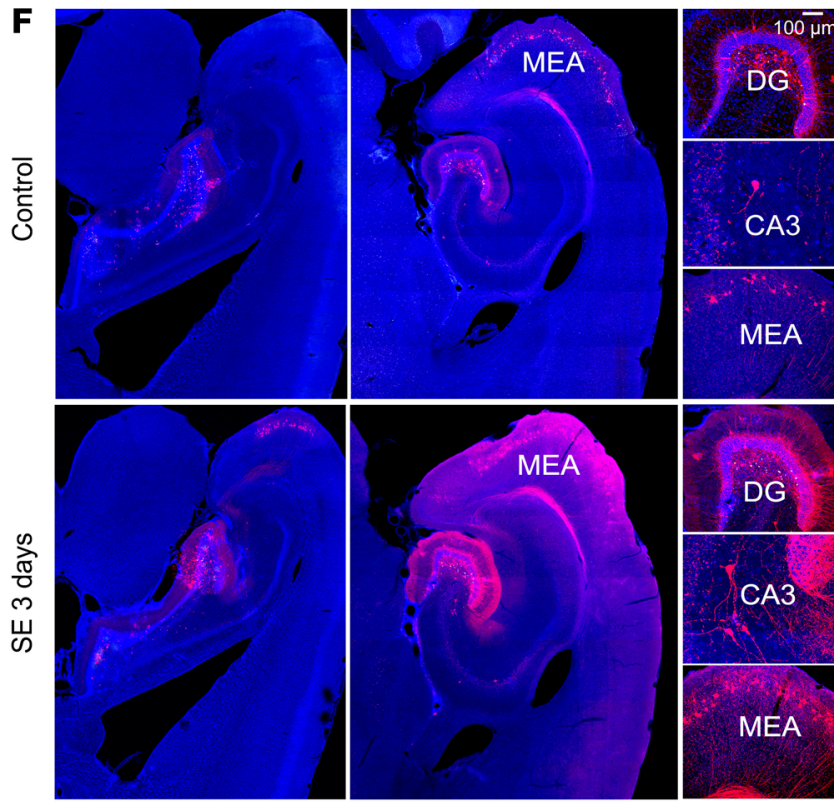
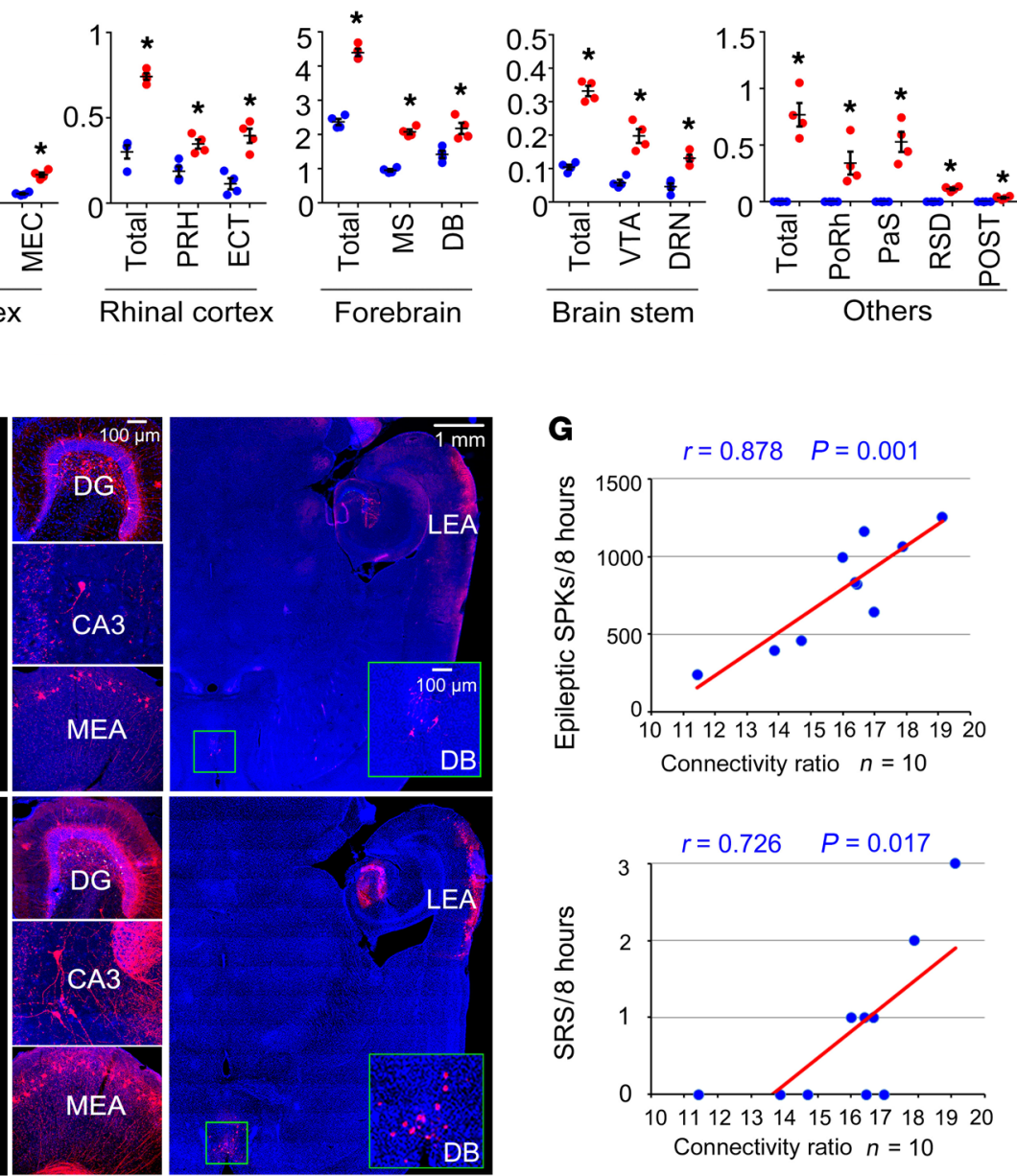

Figure 3. Aberrant integration of hippocampal DGCs contributes to the formation of recurrent excitatory loops. (A) Experimental schematic showing the experimental design for rabies virus-mediated retrograde tracing in epileptic mice. (B-D) The connectivity ratio to input neurons of DGCs born 3 days after SE was significantly increased ( $n=4$ each). (E) Graphs show increases in connectivity ratios between input neurons located in subregions of major brain structures and newborn DCCs ( $n=4$ each). (F) Representative images showing significant changes in the number of input neurons that directly connected to newborn DCCs in epileptic mice. (C) The connectivity ratio was proportional to the severity of epilepsy, as assessed by the frequency of epileptic spikes and SRS $(n=10)$. Data represent mean \pm SEM. ${ }^{*} P<0.05$, Mann-Whitney $U$ tests $(\mathbf{B}-\mathbf{E})$ and Pearson's correlation $(\mathbf{C})$.

Consistent with the observation that abnormal integration of DGCs into neural circuits is dependent upon the age of DGCs relative to SE onset, the abnormal development and migration of DGCs were also dependent upon the age of DGCs at the time of SE. DGCs born 3 days after SE developed abnormally, displaying characteristic morphological impairments, such as hypertrophy, ectopic migration, basal dendrite formation, and axon sprouting to the molecular layer (Figure 2, G-J). However, DGCs born 21 days before SE did not show such structural abnormalities (Fig- ure 2, G, I, and J). These results collectively revealed the critical age window of newborn DGCs relative to the onset of SE, during which abnormally developed DGCs are aberrantly recruited into epileptic neural circuits.

Newborn DGCs contribute to the formation of recurrent excitatory neural circuits. To identify and quantify input neurons that are aberrantly connected to hippocampal newborn DGCs in epileptic mice, RV-Syn-GTRgp was injected into the dentate gyrus 3 days after pilocarpine-induced SE. Two and a half months after SE, 
A

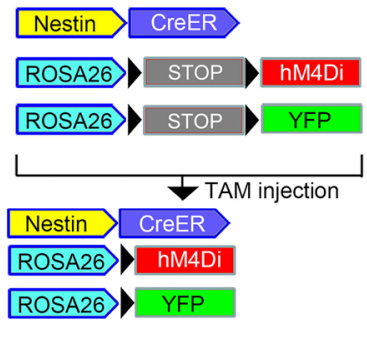

NCE;hM4DI ${ }^{\mathrm{fl} /+}$ or NCE;hM4Di ${ }^{\mid l++} ; \mathrm{YFP}^{\mathrm{H} /+}$

B

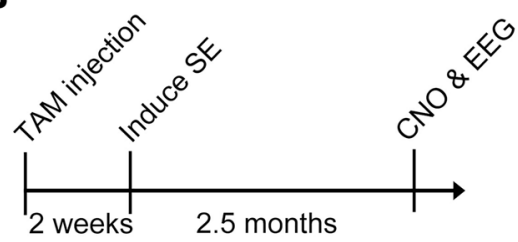

C
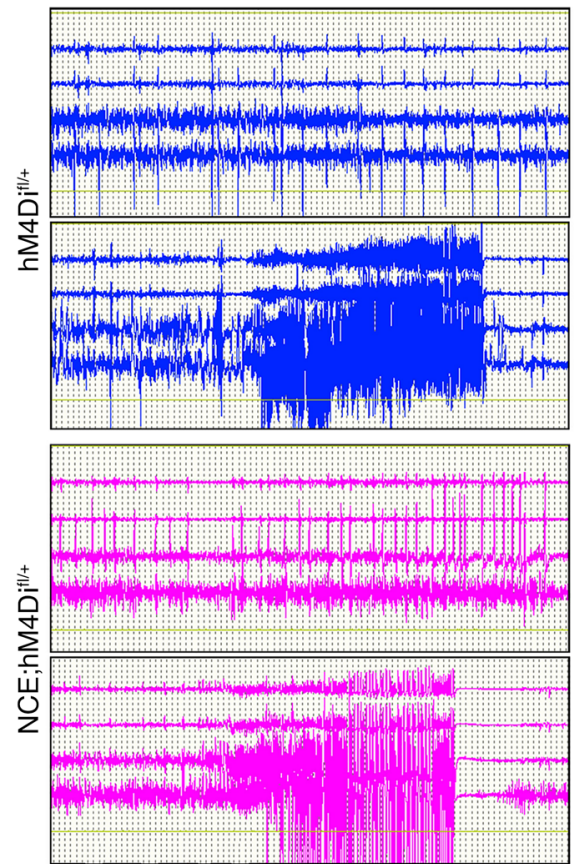
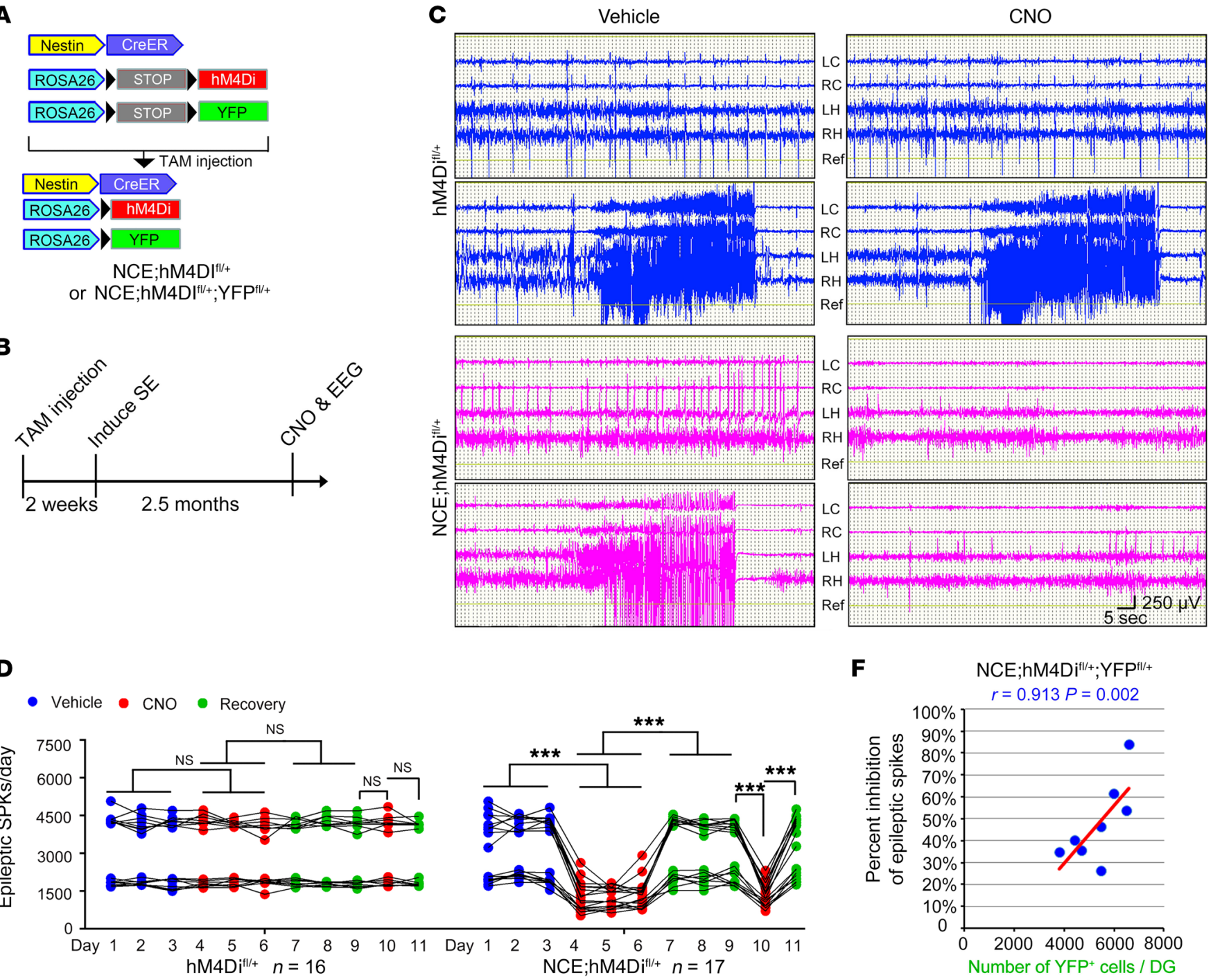

E

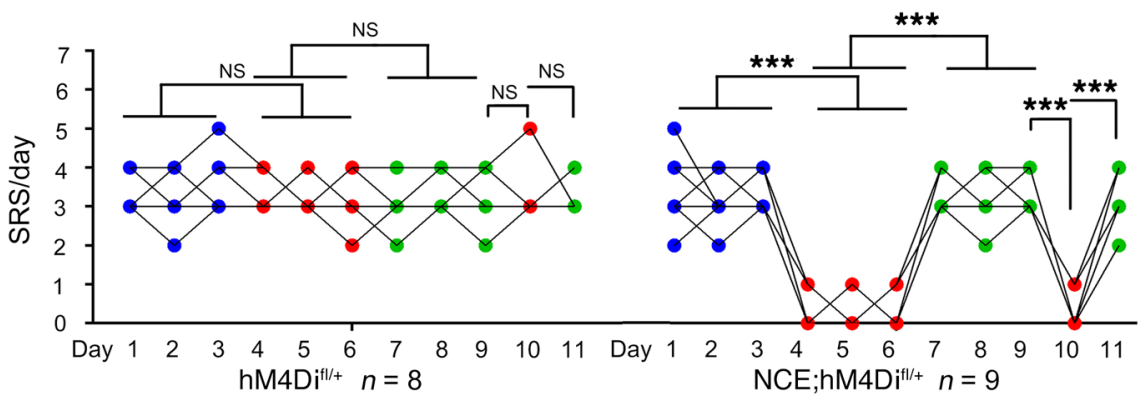

$\mathbf{F}$

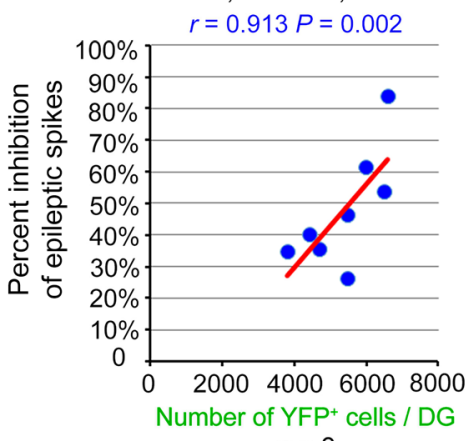

$n=8$

G

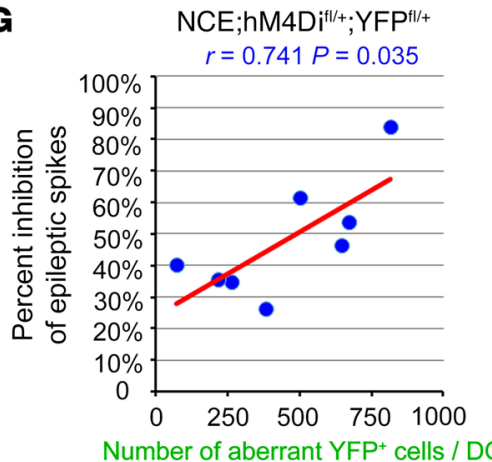

$n=8$

Figure 4. The essential role of hippocampal DGCs in the production of seizures during epilepsy. (A and B) Experimental schematics showing the strategy to selectively suppress the neuronal activity of hippocampal newborn DCCs in NCE; hM4Difl/+ or NCE; hM4Difl/+; $\mathrm{YFP}^{\mathrm{fl} /+}$ mice. (C) Representative EEG recordings show that CNO treatment suppressed epileptic spikes as well as SRS in NCE;hM4Difl/+mice, but not in hM4Difl/+ control mice. (D and E) Epileptic spikes and SRS were quantified during 24 hours of vehicle (days 1-3, blue circles), CNO (days 4-6 and 10, red circles), and recovery without treatment (days 7-9 and 11, green circles). Quantitative results show that CNO treatment effectively reduced the frequency of epileptic spikes $(n=17)$ and SRS ( $n=9)$ only in $\mathrm{NCE} ; \mathrm{hM} 4 \mathrm{Di} \mathrm{f}^{\mathrm{fl} /+}$ mice during epilepsy. ${ }^{* * *} P<0.001$. (F and $\mathbf{G}$ ) Percentage of inhibition of epileptic spikes was proportional to the total number of hM4Diexpressing YFP-positive cells, as well as aberrant hM4Di-expressing YFP-positive cells, in the dentate gyrus. Asterisks in D and E indicate that CNO treatment resulted in a significant reduction in epileptic spikes and SRS compared with vehicle treatment, which returned to basal levels during each recovery period. Two-way RM ANOVA with Bonferroni's multiple comparison tests (D, E) or Pearson's correlation (F, G) were used. 
A

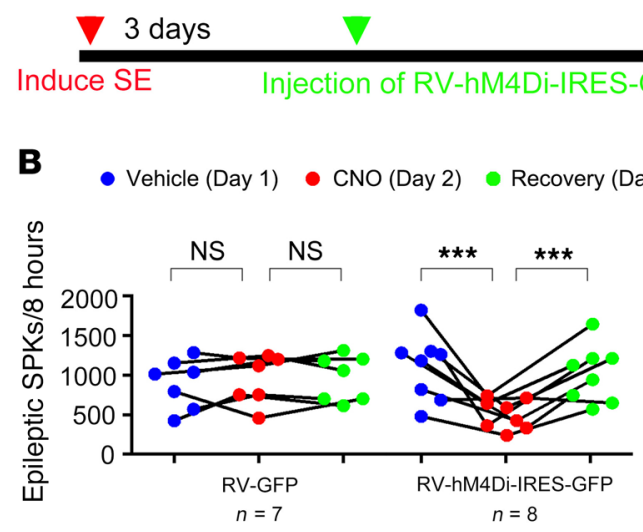

2.5 months

FP

C

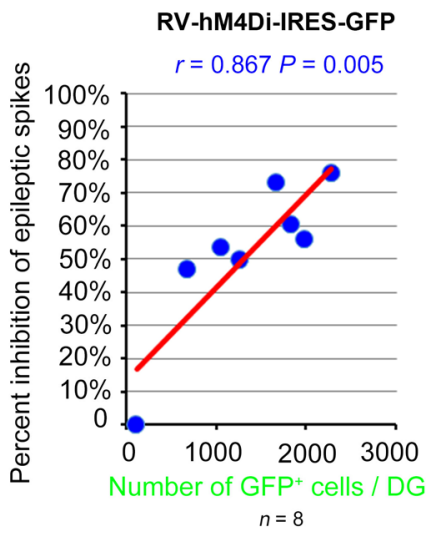

Figure 5. The requirement of adult-born DGCs for the expression of seizures in epileptic mice. (A) Experimental schematic showing strategy for hM4Di receptor expression exclusively in newborn DGCs using RV-hM4Di-IRES-GFP. (B) The process of vehicle treatment (blue circles), CNO treatment (red circles), and recovery without treatment (green circles) was performed on days 1, 2, and 3, respectively. Epileptic spikes and SRS were quantified for 8 hours per day on days 1-3. CNO treatment effectively reduced the occurrence of epileptic spikes and SRS in mice injected with RV-hM4Di-IRES-GFP, but not with RV-GFP. Twenty-four hours after CNO treatment, epileptic spikes and SRS returned to basal levels in RV-hM4Di-IRES-GFP mice. (C) Level of inhibition of epileptic spikes was proportional to the number of hM4Di-expressing YFP-positive cells. ${ }^{* *} P<0.01$; ${ }^{* * *} P<0.001$, 2-way RM ANOVA with Bonferroni's multiple comparison tests (B) and Pearson's correlation (C).

when mice developed spontaneous epilepsy, RbV-EnvA- $\Delta$ RgpMCh was injected, and then neuronal connectivity was analyzed 7 days later (Figure 3A).

In epileptic mice, the connectivity ratio of hippocampal DGCs was globally increased by 2-fold compared with that in control mice (Figure 3, B-D). The increased connectivity of newborn DGCs occurred with input neurons located throughout the brain that normally project to the dentate gyrus, such as the hippocampus, EC, forebrain, and brain stem (Figure 3D). Interestingly, the majority of input neurons that showed increased connectivity to hippocampal newborn DGCs in epileptic mice were excitatory neurons (Figure 3, E and F). Hippocampal newborn DGCs showed increased connectivity with cortical excitatory neurons originating from both the medial (caudomedial EC [CEC] and medial EC [MEC]) and lateral (dorsolateral EC [DLEC], dorsal intermediate EC [DIEC], and ventral intermediate EC [VIEC]) entorhinal areas (Figure 3, E and F). The connectivity ratios of newborn DGCs with input neurons located in the medial septum (MS) and diagonal band (DB) in the forebrain were significantly increased (Figure 3E). Moreover, hippocampal newborn DGCs showed an increased connectivity ratio, with hippocampal excitatory neurons located in the granular layer (GL) of the dentate gyrus and CA3, while the connectivity ratio with hilar neurons was not significantly different in epileptic mice (Figure 3, E and F). The hilus contains both excitatory mossy cells and inhibitory neurons that project to hippocampal newborn DGCs, and the connectivity ratio of hippocampal newborn DGCs with hilar interneurons as well as excitatory neurons did not change in epileptic mice (Supplemental Figure 6). In addition, newborn DGCs form increased connections with input neurons originating from the ventral tegmental area (VTA) and raphe neurons (DRN) located in the brain stem (Figure 3E). A small but significant number of ectopic inputs that were not observed in control mice were identified in epileptic mice. These include neurons located in the postrhinal cortex (PoRH), parasubiculum (PaS), retrosplenial dysgranular cortex
(RSD), and postsubiculum (POST) (Figure 3E). Interestingly, an increased connectivity ratio correlated with the severity of the epilepsy phenotype: the frequencies of epileptic spikes and SRS were proportional to the connectivity ratio of newborn DGCs to afferent input neurons (Figure 3G). These results suggest that cortical as well as intrahippocampal excitatory neurons contribute to the formation of recurrent excitatory neural circuits and that converging excitatory signals are amplified in the dentate gyrus and are propagated to CA3 in epileptic mice.

Hippocampal newborn DGCs are necessary for SRS in epileptic mice. To examine the essential role of hippocampal newborn DGCs in the occurrence of epileptic seizures, neuronal activity of hippocampal newborn DGCs was specifically inhibited by the DREADD method (Figure 4A). First, tamoxifen (TAM) was administered to 6-week-old NCE;hM4Difl/+ mice. This double-transgenic mouse harbors Nestin-CreER and $\mathrm{hM} 4 \mathrm{Di}^{\mathrm{fl} /+}$ alleles and CreER, which is a fusion protein of Cre recombinase and an engineered estrogen receptor expressed exclusively in Nestin ${ }^{+}$neural stem cells. Upon TAM administration, CreER deletes "stop" signals, leading to the expression of the hM4Di receptor in Nestin ${ }^{+}$neural stem cells; all newborn DGCs derived from these Nestin ${ }^{+}$neural stem cells subsequently express the hM4Di receptor. Two weeks later, pilocarpine was administered to induce SE, and the spontaneous development of epilepsy was monitored by video/EEG (Figure 4B). Two and a half months after SE induction, EEG recording showed that both NCE;hM4Difl/+ and control $\left(\mathrm{hM} 4 \mathrm{Di}^{\mathrm{il} /+}\right)$ mice developed spontaneous epilepsy (Figure 4, C-E). On days 1-3, both NCE;hM$4 \mathrm{Di}^{\mathrm{fl} /+}$ and control (hM4 $\left.\mathrm{Di}^{\mathrm{fl} /+}\right)$ mice developed stable epilepsy, displaying consistent epileptic spikes and SRS upon vehicle treatment (Figure 4, D and E). CNO administration on days 4-6 significantly reduced the frequency of epileptic spikes and SRS specifically in NCE;hM4Di ${ }^{\mathrm{il} /+}$ mice, but not in control mice (Figure 4, D and E). On days 7-10, epileptic spikes and SRS returned to basal levels in $\mathrm{NCE} ; \mathrm{hM} 4 \mathrm{Di}^{\mathrm{fl} /+}$ mice and remained at basal levels in control mice (Figure 4, D and E). Epileptic spikes and SRS in NCE;hM4Di ${ }^{\mathrm{fl} /+}$ 
A

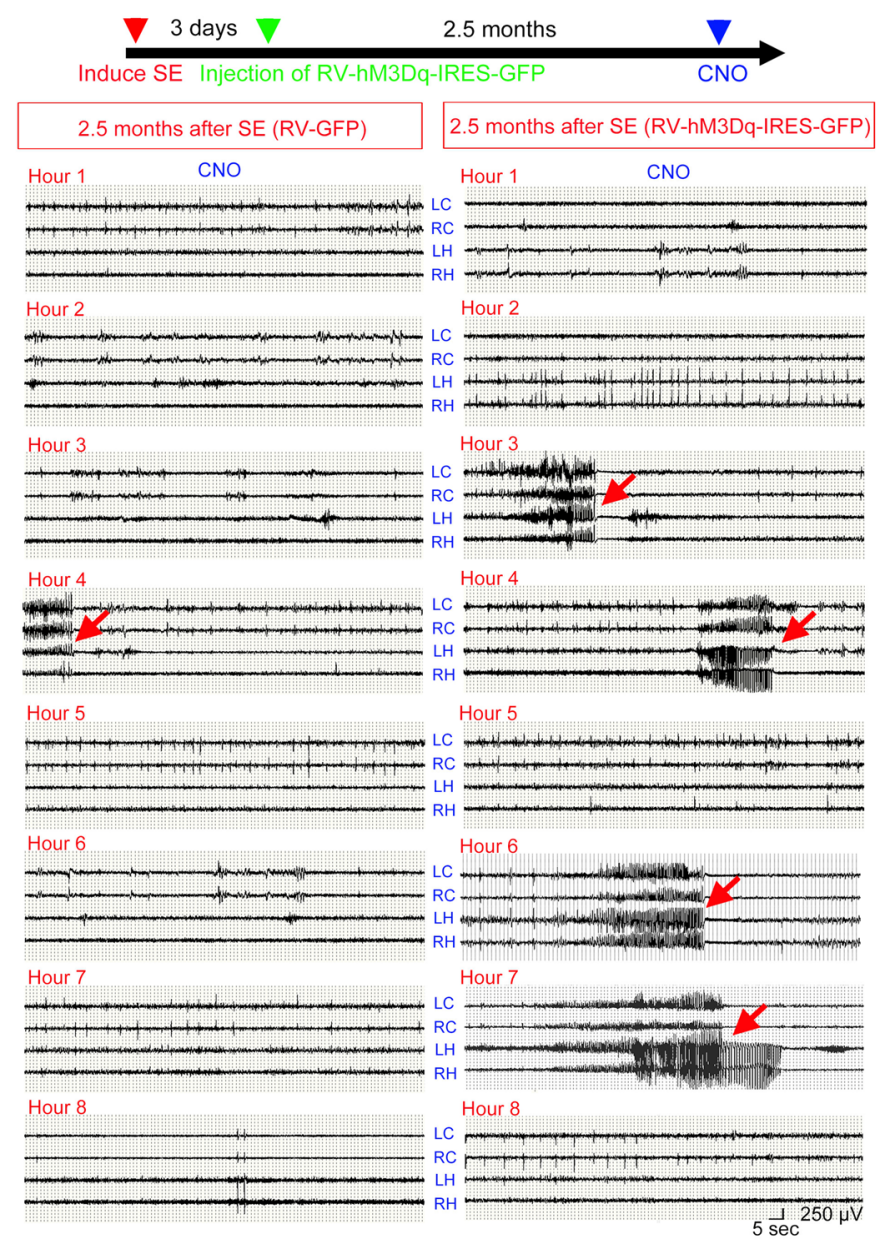

B • Vehicle (Day 1) • CNO (Day 2)
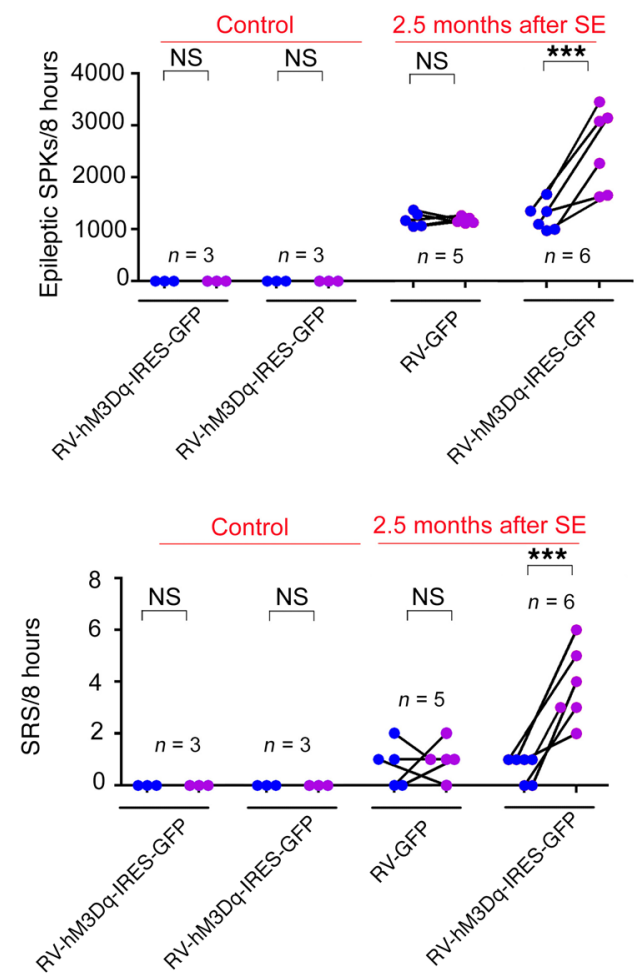

C

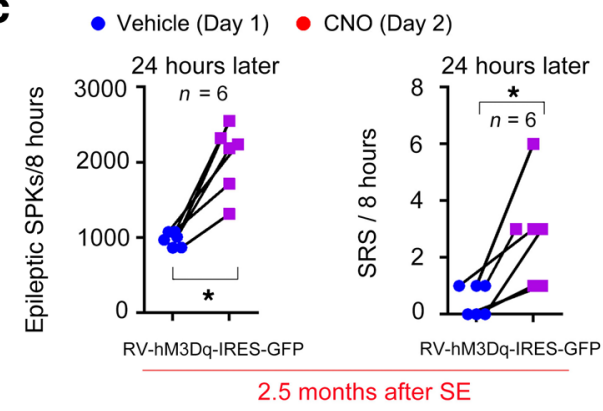

Figure 6. Specific activation of newborn DGCs is sufficient to induce seizures in epileptic mice. (A) Schematic showing the experimental plan to activate hippocampal newborn DGCs in epileptic mice. Representative EEG traces show that specific activation of hippocampal newborn DGCs significantly increased EEG activity and epilepsy frequency. (B) Selective activation of hippocampal newborn DGCs dramatically increased epileptic spikes as well as SRS in epileptic mice. (C) CNO-mediated control of neuronal activity was transiently inducible and reversible. Twenty-four hours after the previous CNOmediated activation of DCCs, repeated CNO administration increased epileptic spikes and SRS. ${ }^{*} P<0.05 ;{ }^{* *} P<0.001,2$-way RM ANOVA with Bonferroni's multiple comparison tests (B) and Wilcoxon's matched-pairs signed rank tests (C).

mice were reduced by injection of $\mathrm{CNO}$ on day 10 and returned to basal levels on day 11 in the absence of CNO (Figure 4, D and E), indicating that specific suppression of hippocampal newborn DGCs reliably reduced seizure expression.

With an independent cohort of NCE;hM4 $4 \mathrm{Di}^{\mathrm{fl} /+} ; \mathrm{YFP}^{\mathrm{fl} /+}$ mice, we repeatedly tested the action of DREADD on seizure expression over a 7-day interval (Supplemental Figure 4, E-H). When mice stably displayed spontaneous epilepsy after SE, CNO administration effectively reduced the frequency of epileptic spikes and SRS in NCE;hM4Di ${ }^{\mathrm{fl} /+} ; \mathrm{YFP}^{\mathrm{fl} /+}$ mice, but not in control mice (Supplemental Figure 4, E and F). Seven days later, administration of $\mathrm{CNO}$ into the same NCE;hM $4 \mathrm{Di}^{\mathrm{fl} /+} ; \mathrm{YFP}^{\mathrm{fl} /+}$ mice reduced epileptic spikes and SRS, indicating that CNO-mediated suppression of hippocampal newborn DGCs is inducible and reversible (Supplemental Figure 4, G and $\mathrm{H}$ ). The number of hM4Di-expressing cells in the dentate gyrus is a critical factor that determines the extent of seizure phenotypes. The degree of inhibition of epileptic spikes was proportional to the number of hM4Di-expressing DGCs and aberrantly developed hM4Diexpressing DGCs (Figure 4, F and G).

Abnormal development and ectopic migration of hippocampal newborn DGCs was evident in NCE;hM4 $\mathrm{Di}^{\mathrm{fl} /+} ; \mathrm{YFP}^{\mathrm{f} /+}$ mice that developed spontaneous epilepsy (Supplemental Figure 7A). The number of c-FOS ${ }^{+}$cells, which is an indicator of activated neurons, was increased in the dentate gyrus of mice that developed spontaneous epilepsy. CNO administration resulted in a trend toward decreased c-FOS ${ }^{+}$cells, but it did not reach statistical significance (Supplemental Figure 7, B and C). However, 
A

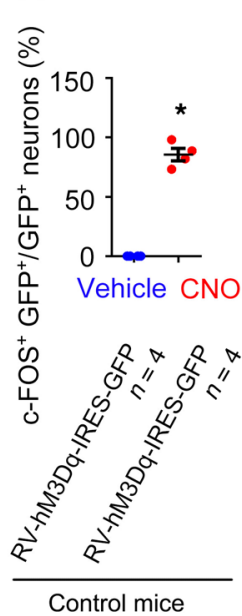

B
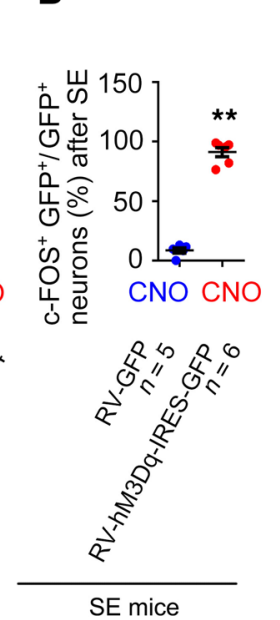

C
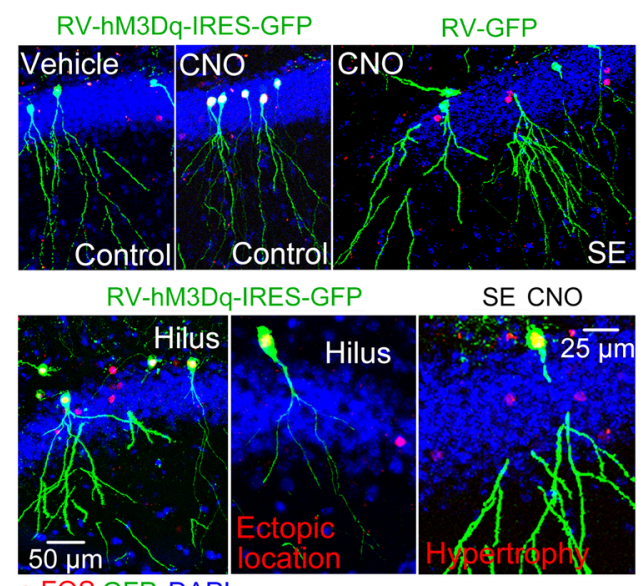

D

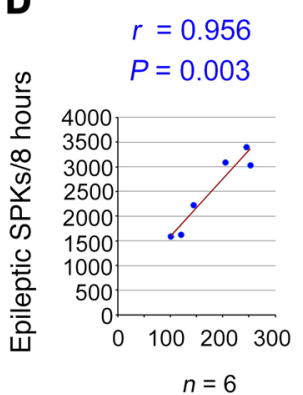

$r=0.873$

$P=0.023$

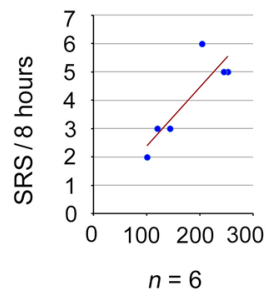

Number of aberrant $\mathrm{GFP}^{+}$neurons/DG

Figure 7. Seizure expression increases proportionally to the number of aberrantly developed hippocampal newborn DCCs. (A and B) Newborn DGCs activated by the CNO-hM3Dq interaction show near complete coexpression with c-FOS in both control (non SE) and epileptic (SE) mice. (C) GFP-labeled hippocampal newborn DGCs develop abnormally in epileptic mice. (D) The number of abnormally developed hippocampal DCCs positively correlates with the severity of epileptic phenotypes, such as epileptic spikes and SRS. Data are shown as mean \pm SEM. ${ }^{*} P<0.05$; ${ }^{*} P<0.01$, Mann-Whitney $U$ tests (A and B) and Pearson's correlation (D).

c-FOS ${ }^{+}$cells within the $\mathrm{YFP}^{+}$cell population were nearly abolished upon CNO administration, indicating specific and effective inhibition of newborn DGCs in an hM4Di receptor activitydependent manner.

The Nestin promoter drove the expression of hM4Di receptors in hippocampal neural stem cells and also in neural stem cells in the subventricular zone that produce olfactory interneurons (Supplemental Figure 8). Although a previous study showed that olfactory interneurons were not likely to be involved in the generation of seizures (7), we directly tested the necessity of hippocampal newborn DGC neuronal activity for seizure expression by expressing hM4Di receptors exclusively in hippocampal newborn DGCs via the retrovirus RV-hM4Di-IRES-GFP (Figure 5). Whole-cell recording showed that CNO treatment effectively reduced the firing rate of hM4Di-expressing hippocampal newborn DGCs (Supplemental Figure 9, A, B, and D). In this experiment, pilocarpine was administered to induce SE. Three days later, RV-hM4Di-IRES-GFP was injected in order to transduce an inhibitory hM4Di and a GFP reporter in hippocampal newborn DGCs. Control mice were injected with a retrovirus that expresses GFP, but not hM4Di (RV-GFP). Two and a half months later, when mice displayed epileptic spikes as well as SRS, CNO was administered (Figure 5A). While the occurrence of epileptic spikes and SRS was not affected in either vehicle-treated control (RV-GFP) or RV-hM4Di-IRES-GFP-injected mice on day 1, CNO injection significantly reduced the frequency of epileptic spikes as well as SRS in hM4Di-expressing mice, but not control mice, on day 2 (Figure 5B). When we measured epileptic spikes and SRS 24 hours after CNO treatment on day 3, the frequencies of both spikes and SRS returned to basal levels, indicating reversible and inducible suppression of the activity of hippocampal newborn DGCs (Figure 5B). The extent of inhibition of epileptic spikes was proportional to the number of hM4Di-expressing DGCs that were born at 3 days after SE induction (Figure 5C).
Activation of hippocampal newborn DGCs is sufficient to induce epileptic spikes and SRS in epileptic mice. Next, we determined whether the activation of hippocampal newborn DGCs is sufficient to induce SRS in epileptic mice. First, pilocarpine was administered to induce SE. Three days later, RV-hM3Dq-IRES-GFP was injected into the hippocampus to induce expression of an excitatory DREADD receptor, hM3Dq, and a GFP reporter in hippocampal newborn DGCs (Figure 6A). Control mice were injected with a retrovirus that expresses GFP (RV-GFP). Whole-cell recording confirmed that CNO treatment significantly increased the firing rate of hM3Dq-expressing hippocampal newborn DGCs (Supplemental Figure 9, A-C). The development of spontaneous epilepsy was monitored by video/EEG. After two and a half months, both groups of mice developed spontaneous epilepsy, as determined by the induction of epileptic spikes and SRS (Figure 6A). In these epileptic mice, CNO-mediated activation of hippocampal DGCs significantly increased the frequency of epileptic spikes and SRS by 2 -fold and 4-fold, respectively, in RV-hM3Dq-IRES-GFP mice, but not in RV-GFP control mice (Figure 6B). At both 24 hours and 7 days after the initial treatment with $\mathrm{CNO}$, vehicle-injected mice showed basal levels of epileptic spikes and SRS. However, administration of CNO dramatically increased epileptic spikes and SRS in RV-hM3Dq-IRES-GFP-injected mice, revealing that the specific activation of hippocampal newborn DGCs is responsible for the induction of epileptic spikes and SRS (Figure 6C and Supplemental Figure 4, I and J). Importantly, CNO administration into RV-hM3Dq-IRES-GFP-injected mice in which SE had not been induced by pilocarpine did not produce epileptic spikes and SRS, indicating that simple activation of neuronal activity of newborn DGCs is not capable of producing epilepsy (Figure 6B).

In contrast to the increased epileptic phenotypes in which DGCs born 3 days after SE were activated, neither epileptic frequency nor SRS was induced when DGCs born 21 days prior to SE were activated (Supplemental Figure 10, A and B). Although a com- 


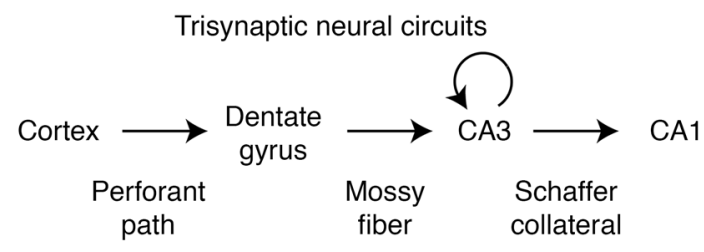

Proepileptic neural circuits

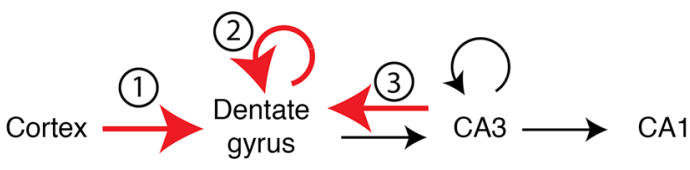

parable number of newborn DGCs expressed hM3Dq receptors in both experiments, DGCs born 21 days prior to SE did not display structural abnormalities (Supplemental Figure 10, C and D).

$\mathrm{CNO}$ treatment efficiently activated hippocampal newborn DGCs, as determined by c-FOS expression in hM3Dq-expressing hippocampal newborn DGCs in both control and epileptic mice (Figure 7, A and B). In epileptic mice, $\mathrm{GFP}^{+}$newborn DGCs born 3 days after $\mathrm{SE}$ showed abnormal development and migration (Figure 7C). Importantly, CNO-mediated induction of epileptic spikes and SRS was proportional to the number of $\mathrm{GFP}^{+}$DGCs that showed abnormal development and aberrant migration (Figure 7D).

\section{Discussion}

Studies from both TLE patients and the pilocarpine-mediated SE animal model have revealed several common key features of TLE (32): (a) the hippocampus as one of the major epileptic loci (33); (b) the presence of a seizure-free latent period between the initial seizure activity (SE) and the development of SRS over time (34); and (c) morphological abnormalities of DGCs characterized by mossy fiber sprouting (10-13), abnormal hilar basal dendrite formation $(15,16)$, and ectopic dispersion and migration $(17,18)$. Interestingly, these structural deficits were restricted to newborn or immature DGCs, while DGCs that had already reached a mature state at the onset of the initial seizure insult were minimally affected (19-21). These observations raised the interesting possibility that seizures primarily influence the development of newborn DGCs and that abnormally formed newborn DGCs aberrantly integrate into proepileptogenic neural networks during the latent period $(7,17,20$, $21,28,35,36)$. While this possibility is intriguing, efforts to assess the contribution of aberrant neural circuits to epilepsy have been hampered by the lack of an adequate tracing system that can be used to identify the exact circuits involved in epilepsy and a method to test the function of the altered neural circuits. In this study, using a rabies virus-mediated retrograde tracing method and DREADD-based chemogenetics that allowed us to map neural circuits and manipulate neuronal activity, we demonstrated an essential role for hippocampal newborn DGCs in the formation of proepileptic neural circuits and the expression of seizures during epilepsy.

The dentate gyrus is located between the EC and CA3. From a computational view, DGCs in the dentate gyrus are the primary projection neurons that relay the information mainly originating in the EC to CA3, enabling spatial and contextual learning and
Figure 8. De novo formation of excessive excitatory networks and recurrent excitatory loops in the hippocampus of epileptic mice. In the normal hippocampus (top panel), input neurons located in the EC send their excitatory projections to the dentate gyrus via the perforant pathway. DGCs in the dentate gyrus project their axons (mossy fibers) to CA3 pyramidal neurons. Pyramidal neurons are interconnected, forming recurrent networks in CA3, and project to CA1 neurons via Schaffer collaterals. This unidirectional excitatory neuronal network, cortex $\rightarrow$ dentate gyrus $\rightarrow$ CA $\rightarrow$ CA1, has been referred to as trisynaptic connections. In epileptic mice (lower panel), excitatory inputs from the $\mathrm{EC}$ to the dentate gyrus are increased, reinforcing the convergence of excitatory signals to the dentate gyrus (no. 1). In addition, de novo formation of recurrent excitatory loops within the dentate gyrus (no. 2), as well as between the dentate gyrus and CA3 (no. 3), facilitates the production, amplification, and propagation of excitatory signals and may be responsible for synchronous discharges in the hippocampus.

memory function (9). Physiologically, DGCs are excitatory neurons: DGCs receive excitatory afferent inputs from the EC and produce and relay excitatory output to CA3. Although substantial excitatory input to DGCs is expected to produce a significant amount of excitatory drive, hippocampal DGCs are sparsely activated due to feedforward and feedback inhibition (9). This observation suggests that the dentate gyrus functions as a "gate" that prevents excessive excitatory signals from propagating to CA3 and CA1 (37) and that the disruption of this dentate gate may underlie epilepsy (38-40). Indeed, our study clearly provides evidence that abnormally developed and aberrantly integrated hippocampal newborn DGCs contribute to functional disruption of the dentate gate, resulting in the dentate gyrus producing excessive excitatory signals and relaying them first to CA3, and subsequently to CA1, in epileptic mice.

Our rabies virus-mediated mapping studies revealed that hippocampal newborn DGCs play a critical role in the occurrence of seizures by forming de novo excitatory neural circuits as well as excitatory recurrent loops in the hippocampus (Figure 8). Among these, 3 major alterations in neural circuits are expected to be critical for seizure expression. First, in epileptic mice, the connectivity ratio of excitatory-to-inhibitory connections to newborn DGCs was substantially increased. The connectivity of newborn DGCs was also globally increased, with excitatory neurons originating from various brain structures, such as the EC, forebrain, brain stem, and hippocampus, while the connectivity ratio of newborn DGCs with inhibitory neurons was not significantly altered. This convergence of excitatory projections to newborn DGCs reinforces neural circuits that allow excessive excitatory signals to enter the dentate gyrus (Figure 8). Second, in the normal hippocampus, there are few direct interconnections between DGCs. However, our tracing results showed that interconnections between hippocampal newborn DGCs and neighboring DGCs were significantly increased in epileptic mice (Figure 3E). This de novo formation of recurrent excitatory loops within the dentate gyrus is caused by abnormally developed and integrated newborn DGCs. For example, ectopic hilar basal dendrites and abnormally migrating newborn DGCs are perfectly positioned to receive excitatory mossy fiber innervations. Simultaneously, axons of hippocampal newborn DGCs sprouting into the inner molecular layer, together with axonal reorganization of mature DGCs, form excitatory synapses onto neighboring DGCs (14). In addition, mossy cells in the hilus 
are excitatory neurons that receive input from DGCs and send excitatory output to DGCs, and extensive loss of mossy cells is associated with SE (41). Our observation that the connectivity with mossy cells did not change suggests a net increase in connectivity of newborn DGCs with surviving excitatory mossy cells. Thus, de novo formation of recurrent excitatory loops within the dentate gyrus may underlie the production and amplification of excessive excitatory signals and synchronous discharge in the dentate gyrus of epileptic mice (Figure 8). Third, DGCs normally project to CA3 pyramidal neurons, forming characteristic unidirectional excitatory connections between the dentate gyrus and CA3. However, our tracing results showed that newborn DGCs received significantly increased projections from CA3 pyramidal neurons, forming de novo reciprocal connections between the dentate gyrus and CA3 in epileptic mice. Increased reciprocal connections between newborn DGCs and CA3 pyramidal neurons facilitate the propagation of excessive excitatory signals to CA3, synchronous discharge between the dentate gyrus and CA3, and "back propagation" from CA3 to the dentate gyrus $(42,43)$ (Figure 8 ). Our mapping data reveal the anatomical route through which back propagation, which is a seizure-characteristic propagation from CA3, transmits directly from CA3 to the dentate gyrus. Together, our mapping results reveal a critical role for newborn DGCs in the de novo formation of recurrent excitatory circuits and loops that are responsible for the production, amplification, and propagation of excitatory signals in epileptic brains.

Using an inducible and reversible manipulation of neuronal activity in hippocampal newborn DGCs, our study revealed an essential role for hippocampal newborn DGCs in the production of epileptic spikes and seizures during epilepsy. Specific silencing of hippocampal DGCs significantly reduced epileptic spikes and SRS, revealing a requirement of hippocampal DGCs for seizure expression during epilepsy (Figure 4, D-E, and Figure 5B). Conversely, specific activation of hippocampal DGCs dramatically increased the frequency of epileptic spikes as well as SRS, indicating that increased activity of hippocampal DGCs is sufficient to activate epileptic neural circuits. These results showed that hippocampal newborn DGCs are responsible for remodeling hippocampal neural circuits and that these altered neural circuits underlie induction of seizures during epilepsy. To determine the essential role of hippocampal newborn DGCs, ablation of neurogenesis has been widely used $(6,8,20)$. However, it has been reported that ablation of the neuronal population may induce rewiring of the neuronal connectivity of existing neurons $(44,45)$. This observation raised a concern as to whether the reduced SRS observed in ablation studies was caused by the depletion of hippocampal DGCs or by network rearrangement secondary to the loss of hippocampal DGCs. In addition, it is not possible to assess whether hippocampal newborn DGCs are directly involved in seizure production in epileptic mice when newborn neurons are ablated. In order to rule out potential network remodeling associated with ablation, we specifically manipulated the activity of hippocampal newborn DGCs in an inducible and reversible manner only in mice that actually developed spontaneous epilepsy. Any changes, including neural circuit formation, were not interrupted during epileptogenesis and were maintained intact, while only the neuronal activity of hippocampal newborn DGCs was controlled in an inducible and reversible manner. As a result, our study clearly demonstrated the direct contribution of the activity of hippocampal newborn DGCs to seizure expression in a pilocarpine-induced TLE mouse model.

In summary, we used a mapping and neuronal manipulation method with cell type-specific resolution to provide evidence supporting the long-standing hypothesis that hippocampal newborn DGCs are necessary and sufficient for the expression of seizures during epilepsy. In addition, we showed that hippocampal newborn DGCs are an essential node in proepileptic neural circuits, raising the possibility that hippocampal neurogenesis may be an effective target for epilepsy inhibition. Future studies to determine the specific age of newborn DGCs relative to SE induction will be critical for the targeted development of new therapies to treat epilepsy.

\section{Methods}

Mice. Six- to nine-week-old male mice were used in this study. POMCCre (stock 005965) and C57BL/6 (stock 000664) mice were purchased from The Jackson Laboratory. Nestin-CreER and PDi (hM4Dit/il) mice were provided by Amelia Eisch (Department of Anesthesiology and Critical Care, University of Pennsylvania, Philadelphia, Pennsylvania, USA) (46) and in house (24). All transgenic mice were backcrossed to C57BL/6 for more than 10 generations. Mice were housed with a 12-hour light/12-hour dark cycle (light on at 6:00 am and off at 6:00 pm), with food and water available ad libitum.

Data acquisition and analysis. To reduce bias in data collection, analysis, and reporting, experimental conditions and subjects were randomly coded, and the investigators were blinded to treatment conditions and codes until all data had been collected and analyzed.

TAM and CNO treatment to activate Cre-ER and DREADD receptors. CNO was purchased from Sigma-Aldrich. CNO $(10 \mathrm{mg} / \mathrm{kg}$ or $1 \mathrm{mg} / \mathrm{kg})$ was i.p. injected to activate hM4Di and hM3Dq DREADD receptors, respectively. To study its long-term effects, CNO $(10 \mathrm{mg} / \mathrm{kg}$, i.p., every 8 hours for 3 days) was continuously administered for 3 days. DMSO $(0.5 \%)$ in saline was used as a vehicle. To express hM4Di receptors and the YFP reporter in newborn DGCs in Nestin-CreER;hM4 $\mathrm{Di}^{\mathrm{fl} /+} ; \mathrm{YFP}^{\mathrm{fl} /+}$ transgenic mice, TAM $(180 \mathrm{mg} / \mathrm{kg}$, i.p., dissolved in a 1:10 mixture of ethanol and sunflower oil, Sigma-Aldrich) was administered daily for 5 days when the mice were 6 weeks old.

$S E$ induction by pilocarpine. SE was induced when mice were 8 weeks old. After $N$-methyl scopolamine ( $1 \mathrm{mg} / \mathrm{kg}$, Sigma-Aldrich) was injected i.p., pilocarpine $(290 \mathrm{mg} / \mathrm{kg}$, Sigma-Aldrich) was then injected i.p. SE was terminated by injection of diazepam $(10 \mathrm{mg} /$ kg, i.p., Hospira) 3 hours after SE induction. During SE induction, animals gradually displayed seizure behaviors, starting from milder conditions, such as immobility (stage 1) and forelimb and/or tail extension (stages 2-3), to severe stages, showing continuous rearing and falling (stages 4-5) and tonic-clonic seizures (stage 6). Coincident with behavioral development into SE, EEG first showed epileptic spikes, defined by a field potential that was 2-fold greater than the basal potential, followed by seizure activities, which were defined as continuous epileptic spikes longer than 10 seconds. In this project, pilocarpine was injected into a total of 238 mice to induce epilepsy. Of these, 64 mice (26.89\%) and 47 mice (19.75\%) were excluded due to lethality and absence of spikes, respectively, and a total of 127 mice (53.36\%) were included in the major experiments. Among these 127 mice that displayed epileptic spikes, 69 subsequently exhibited SRS. 
Detailed information for each experiment is summarized in Supplemental Table 1. All mice that displayed epileptic spikes and SRS retained these epileptic phenotypes, and the frequency of spikes and SRS gradually increased over time.

Virus production. The production of retrovirus and rabies virus was previously described (22). Retroviral (RV-SYN-GTRgp) vectors were generated to express hGFP, TVA, and Rgp under the control of the synapsin promoter. Lipofectamine 2000 (Invitrogen) was used to produce retrovirus by transient transfection of the RV-SYN-GTRgp vector (7.5 $\mu \mathrm{g})$, as well as the helper vectors CMV-GP $(5 \mu \mathrm{g})$ and CMV-VSVG $(2.5$ $\mu \mathrm{g}$ ), into $60 \%$ confluent $293 \mathrm{~T}$ cells grown in $10 \mathrm{~cm}$ plates. Virus-containing supernatant was harvested 36 hours later and ultracentrifuged. The final titers were estimated to be approximately $10^{8} \mathrm{CFU} /$ $\mathrm{ml}$, as determined by the infection of $293 \mathrm{~T}$ cells with serially diluted virus. The production of RbV-EnvA- $\triangle \mathrm{Rgp}-\mathrm{MCh}$ was carried out as described previously (47). Briefly, the endogenous rabies virus glycoprotein was replaced with an mCherry reporter gene $(\Delta \mathrm{gp}-\mathrm{mCher}-$ ry), and this rabies virus vector was pseudotyped with EnvA receptor (RbV-EnvA- $\triangle$ Rgp-MCh). The titer of rabies virus was estimated to be approximately $10^{8} \mathrm{CFU} / \mathrm{ml}$, and virus was diluted to approximately $5 \times$ $10^{7} \mathrm{CFU} / \mathrm{ml}$ for use. For the production of RV-hM3Dq-IRES-GFP and RV-hM4Di-IRES-GFP, the open reading frames of hM3Dq and hM4Di were obtained from pcDNA5/FRT-HA-hM3Dq (Addgene, catalog 45547) and pcDNA5/FRT-HA-hM4Di (Addgene, catalog 45548) and used to produce RV-hM3Dq-IRES-GFP and RV-hM4Di-IRES-GFP. $\mathrm{RV}-\mathrm{GFP}$ was also produced as a control. The titers of both viruses were estimated to be approximately $1.2 \times 10^{8} \mathrm{CFU} / \mathrm{ml}$.

Surgical procedures. The surgical procedure was performed as described previously (22), with additional changes to the injection coordinates. Mice were anesthetized with a mixture of ketamine (100 $\mathrm{mg} / \mathrm{kg}$, ACE Surgical Supply Co.) and xylazine (10 mg/kg, SigmaAldrich). Stereotactic surgery was performed to deliver a volume of 1 $\mu$ l of RV-Syn-GTRgp into the right side of the dentate gyrus using the following coordinates: anterior-posterior $(\mathrm{AP})=-1.35 \mathrm{~mm}$; medial-lateral $(\mathrm{ML})=1.3 \mathrm{~mm}$; dorsal-ventral $(\mathrm{DV})=2.35 \mathrm{~mm}, \mathrm{AP}=-2.5 \mathrm{~mm}$; $\mathrm{ML}=2.65 \mathrm{~mm} ; \mathrm{DV}=3.4 \mathrm{~mm}$, and $\mathrm{AP}=-2.25 \mathrm{~mm} ; \mathrm{ML}=2.45 \mathrm{~mm} ; \mathrm{DV}$ $=4.75 \mathrm{~mm}$. The rabies virus RbV-EnvA- $\Delta$ Rgp-MCh $(1 \mu \mathrm{l})$ was delivered into the same locations 1 or 2.5 months later. When hM3Dq or hM4Di receptors were expressed using RV, RV-hM3Dq-IRES-GFP or RV-hM4Di-IRES-GFP was bilaterally injected into the dentate gyrus. A motorized stereotaxic injector (Stoelting, 53311) was used to infuse virus into the hippocampus at a rate of $0.2 \mu \mathrm{l} / \mathrm{min}$.

IHC. Mice were deeply anesthetized with a mixture of ketamine (100 mg/kg, ACE Surgical Supply Co., 950-9508) and xylazine (10 $\mathrm{mg} / \mathrm{kg}, \mathrm{X1126}$, Sigma-Aldrich) and perfused intracardially with saline followed by $4 \%$ paraformaldehyde (PFA) (16005, Sigma-Aldrich). Brains were removed, post-fixed overnight in $4 \%$ PFA, and cryopreserved in $30 \%$ sucrose. IHC was carried out on $40 \mu \mathrm{m}$ free-floating sections as described (22). Primary antibodies were as follows: GFP (chicken; 1:500, catalog 1020, Aves), mCherry (rabbit; 1:500, catalog ab167453, DAKO), c-FOS (rabbit; 1: 100, catalog SYSY226003, Synaptic Systems, The Antibody Company), calretinin (rabbit; $1: 500$, catalog CR7697, Swant Inc.), GABA (rabbit; 1:200, catalog PA5-32241, Thermo Fisher Scientific), NPY (rabbit; 1:400, catalog ab10980, Abcam), and parvalbumin (rabbit; 1:500, catalog PV27, Swant Inc.). Nuclei were visualized with DAPI. Cy3-, Cy5-, or FITC-conjugated secondary antibodies (1:200; Jackson ImmunoResearch) were used in this study.
Cell counting. To analyze the distribution of traced cells throughout the brain, whole brains were cut horizontally at a thickness of 40 $\mu \mathrm{m}$. The numbers of $\mathrm{mCherry}^{+}$cells (traced, afferent, or input cells) or cells double-labeled with hGFP and MCh (starter cells) were quantified in all sections. Only the mice that displayed a stage 4 or higher seizure phenotype during SE induction were used to study anatomical connectivity. To analyze and quantify input neurons and c-FOS ${ }^{+}$cells, IHC and quantification were carried out in a 1:6 series of horizontal sections. All positive cells were counted for the given sections, and the total number was then calculated by multiplying by the interval factor 6. All fluorescence images were collected using laser confocal microscopy (SP5, Leica) and further processed in Adobe Photoshop. For c-FOS ${ }^{+}$cell counting, animals were sacrificed within 90 to 120 minutes after CNO treatment. During the experiment, CNO was injected into individual mice at 15-minute time intervals. In this manner, all mice could be perfused within a time frame of 90 to 120 minutes after CNO administration. Control mice received vehicle instead of CNO and were treated in the same manner.

Implantation of electrodes for EEG. Animals were anesthetized with a mixture of ketamine $(100 \mathrm{mg} / \mathrm{kg}$, ACE Surgical Supply Co.) and xylazine (10 mg/kg, Sigma-Aldrich) and placed in a stereotactic apparatus (David Kopf Instruments). For hippocampal recordings, bipolar twisted stainless-steel electrodes $(0.2 \mathrm{~mm}$ in diameter; PlasticOne) were placed bilaterally in the hippocampi (left hippocampus [LH]; right hippocampus [RH]; $2.75 \mathrm{~mm}$ posterior to the bregma, $2.45 \mathrm{~mm}$ lateral to the midline, and $2.85 \mathrm{~mm}$ below the dura). Stainless-steel screws (MX-0090-2; PlasticOne) were placed epidurally and bilaterally over the frontal cortices (left frontal [LF]; right frontal [RF], $0.5 \mathrm{~mm}$ posterior to the bregma, 2.45 $\mathrm{mm}$ lateral to the midline). An additional screw was placed just to the right of the frontal sinus and served as a referential electrode (REF). The electrodes were then connected to a plastic plug (SMB06V-BC, Solderless Terminal), which was fixed to the skull with dental cement (hygenic repair resin; Henry Schein Inc.). Mice were left unrestrained for 24-72 hours to allow for recovery from surgery before further manipulation and prolonged EEG monitoring were initiated. Digital EEG recordings were performed using a Vangard system (Lamont). The recording filter settings were as follows: low-frequency filter of 1 and high-frequency filter of $70 \mathrm{~Hz}$. Video/ EEG was continuously monitored for 16 hours per day, beginning at the onset of the dark cycle of the mouse facility $(6: 00 \mathrm{pm})$ for 2 or 3 days per week for 13 weeks. For experiments, including CNO treatment (Supplemental Figure 4, A-D; Figure 4, F and G; Figure 5, $\mathrm{G}$ and $\mathrm{H}$; and Figure 6, B and C), on day 1, vehicle was injected at the onset of the dark cycle, and EEG was recorded. On day 2, CNO was injected into the same mice at the beginning of the dark cycle, and EEG was monitored. To test the reversibility of CNO-mediated manipulation of neuronal activity, the procedures performed on days 1 and 2 were repeated in some mice. Only the first 8 hours of EEG recording were used for data analysis, based on our assessment of the effects of CNO in the system. For experiments described in Figure 1, D and E, and Figure 4, D and E, video/EEG was continuously monitored for 24 hours per day for 9 or 11 days. Vehicle or CNO $(10 \mathrm{mg} / \mathrm{kg}$, i.p.) was injected into the same mice at 8-hour intervals. All data collected for 24 hour EEG recording from each day were used for analysis. Implanted recording electrodes were connected to pendulous electroslip rings, which permitted acquisition of real- 
time noise and artifact-free EEGs when the mice were moving and during seizures. During recording, mice were unrestrained in the monitoring boxes, with free access to food and water ad libitum.

Electrophysiology. Brain slices were prepared with a Vibratome (VT1200s, Leica) in ice-cold, oxygenated artificial CSF (aCSF) containing $110 \mathrm{mM} \mathrm{NaCl}, 0.5 \mathrm{mM} \mathrm{CaCl}_{2}, 2.5 \mathrm{mM} \mathrm{KCl}, 7 \mathrm{mM} \mathrm{MgCl}, 1.3$ $\mathrm{mM} \mathrm{NaH} \mathrm{PO}_{4}, 1.3 \mathrm{mM}$ Na-ascorbate, $0.6 \mathrm{~m} \mathrm{M}$ Na-pyruvate, $25 \mathrm{mM}$ $\mathrm{NaHCO}_{3}$, and $20 \mathrm{mM}$ glucose and then incubated in warm oxygenated $\operatorname{aCSF}\left(34^{\circ} \mathrm{C}\right)$ for 1 hour. Brain slices $(350 \mu \mathrm{m})$ showing the hippocampal region were transferred into the recording chamber and superfused $(2 \mathrm{ml} / \mathrm{min})$ with oxygenated aCSF at room temperature $\left(22-25^{\circ} \mathrm{C}\right)$. Whole-cell patch recordings were performed with a computer-controlled amplifier (MultiClamp 700B, Molecular Devices). The pipettes for cell-attached recording (3-4 M $\Omega$ ) contained bath solution, and the seal resistance was approximately $200 \mathrm{M} \Omega$. GFP-positive neurons were targeted in the DG area under the visual guidance of green fluorescent signals using an upright microscope (Examiner Z1, Zeiss). CNO $(10 \mu \mathrm{M})$ dissolved in aCSF was released to the recording area with small pressure using an 8-channel drug-delivery system (MPS-1, Inbio Life Science Instrument).

Statistics. Statistical analyses were performed using commercial software (GraphPad Prism; GraphPad Software Inc.) and SPSS (IBM). For all results, the significance threshold was placed at $\alpha=0.05$, and corrections for multiple comparisons are reflected in $P$ values. Correlational analyses were performed by using Pearson's correlation tests in SPSS. To compare 2 independent groups with respect to a numeric outcome, Mann-Whitney $U$ tests were used. To compare 2 matched samples for repeated numeric measurements, Wilcoxon's signed-rank tests were used. To compare 3 or more independent groups with respect to a numeric outcome, Kruskal-Wallis tests with Dunn's multiple comparison corrections were used. To compare the effect of 2 factors with respect to a numeric outcome, we used 2-way ANOVA Bonferroni's multiple comparison corrections. To repeatedly measure the effect of the DREADD method on the seizure phenotypes in the same subjects and to compare the mean differences between groups, 2-way repeated measures (RM) ANOVA with Bonferroni's multiple comparison corrections were used. Data are represented as mean \pm SEM.

Study approval. All procedures involving animals were approved by the Institutional Animal Care and Use Committee (IACUC) of Nanjing Medical University and Cleveland Clinic. All experiments were conducted in accordance with the principles and procedures outlined in the NIH Guide for the Care and Use of Laboratory Animals (National Academies Press, 2011).

\section{Author contributions}

HS and QGZ designed all experiments. QGZ, ADN, DL, and EJR performed the experiments. SMD and IMN provided mice and resources for EEG. JZ performed the electrophysiology. ASN assisted statistical analysis. HS and QGZ analyzed the data and wrote the manuscript.

\section{Acknowledgments}

We thank Edward M. Callaway for providing the engineered rabies virus (Systems Neurobiology Laboratory, Salk Institute, La Jolla, California, USA). We also thank Christopher L. Nelson for editorial support. This work was supported by a grant from the National Natural Science Foundation of China (81571269 to QGZ) and by the National Institute of Alcohol Abuse and Alcoholism (R01AA022377 to HS), the Whitehall Foundation (to HS), and the Hartwell Foundation (to HS).

Address correspondence to: Hoonkyo Suh, Department of Neurosciences, NC 30, Cleveland Clinic, Cleveland, Ohio 44195, USA. Phone: 216.444.5895; Email: Suhh2@ccf.org.
1. French JA, et al. Characteristics of medial temporal lobe epilepsy: I. Results of history and physical examination. Ann Neurol. 1993;34(6):774-780.

2. Williamson PD, et al. Characteristics of medial temporal lobe epilepsy: II. Interictal and ictal scalp electroencephalography, neuropsychological testing, neuroimaging, surgical results, and pathology. Ann Neurol. 1993;34(6):781-787.

3. Engel J. Etiology as a risk factor for medically refractory epilepsy: a case for early surgical intervention. Neurology. 1998;51(5):1243-1244.

4. Kwan P, Brodie MJ. Early identification of refractory epilepsy. N Engl J Med. 2000;342(5):314-319.

5. Chang BS, Lowenstein DH. Epilepsy. N Engl J Med. 2003;349(13):1257-1266.

6. Hosford BE, Liska JP, Danzer SC. Ablation of newly generated hippocampal granule cells has disease-modifying effects in epilepsy. JNeurosci. 2016;36(43):11013-11023.

7. Pun RY, et al. Excessive activation of mTOR in postnatally generated granule cells is sufficient to cause epilepsy. Neuron. 2012;75(6):1022-1034.

8. Cho KO, et al. Aberrant hippocampal neurogenesis contributes to epilepsy and associated cognitive decline. Nat Commun. 2015;6:6606.

9. Amaral DG, Scharfman HE, Lavenex P. The dentate gyrus: fundamental neuroanatomical organization (dentate gyrus for dummies). Prog Brain Res. 2007;163:3-22.
10. Sutula T, He XX, Cavazos J, Scott G. Synaptic reorganization in the hippocampus induced by abnormal functional activity. Science. 1988;239(4844):1147-1150.

11. Tauck DL, Nadler JV. Evidence of functional mossy fiber sprouting in hippocampal formation of kainic acid-treated rats. J Neurosci. 1985;5(4):1016-1022.

12. Sutula TP, Dudek FE. Unmasking recurrent excitation generated by mossy fiber sprouting in the epileptic dentate gyrus: an emergent property of a complex system. Prog Brain Res. 2007;163:541-563.

13. Scharfman HE, Sollas AL, Berger RE, Goodman JH. Electrophysiological evidence of monosynaptic excitatory transmission between granule cells after seizure-induced mossy fiber sprouting. J Neurophysiol. 2003;90(4):2536-2547.

14. Althaus AL, Zhang H, Parent JM. Axonal plasticity of age-defined dentate granule cells in a rat model of mesial temporal lobe epilepsy. Neurobiol Dis. 2016;86:187-196.

15. Ribak CE, Tran PH, Spigelman I, Okazaki MM, Nadler JV. Status epilepticus-induced hilar basal dendrites on rodent granule cells contribute to recurrent excitatory circuitry. JComp Neurol. 2000;428(2):240-253.

16. Buckmaster PS, Dudek FE. In vivo intracellular analysis of granule cell axon reorganization in epileptic rats. J Neurophysiol. 1999;81(2):712-721. 17. Scharfman HE, Goodman JH, Sollas AL. Gran- ule-like neurons at the hilar/CA3 border after status epilepticus and their synchrony with area CA3 pyramidal cells: functional implications of seizure-induced neurogenesis. J Neurosci. 2000;20(16):6144-6158.

18. Houser CR, Swartz BE, Walsh GO, DelgadoEscueta AV. Granule cell disorganization in the dentate gyrus: possible alterations of neuronal migration in human temporal lobe epilepsy. Epilepsy Res Suppl. 1992;9:41-48; discussion 48.

19. Jessberger S, Römer B, Babu H, Kempermann G. Seizures induce proliferation and dispersion of doublecortin-positive hippocampal progenitor cells. Exp Neurol. 2005;196(2):342-351.

20. Kron MM, Zhang H, Parent JM. The developmental stage of dentate granule cells dictates their contribution to seizure-induced plasticity. JNeurosci. 2010;30(6):2051-2059.

21. Walter C, Murphy BL, Pun RY, Spieles-Engemann AL, Danzer SC. Pilocarpine-induced seizures cause selective time-dependent changes to adult-generated hippocampal dentate granule cells. JNeurosci. 2007;27(28):7541-7552.

22. Vivar C, et al. Monosynaptic inputs to new neurons in the dentate gyrus. Nat Commun. 2012;3:1107.

23. Alexander GM, et al. Remote control of neuronal activity in transgenic mice expressing evolved G protein-coupled receptors. Neuron. 
2009;63(1):27-39.

24. Ray RS, et al. Impaired respiratory and body temperature control upon acute serotonergic neuron inhibition. Science. 2011;333(6042):637-642.

25. Garner AR, et al. Generation of a synthetic memory trace. Science. 2012;335(6075):1513-1516.

26. McHugh TJ, et al. Dentate gyrus NMDA receptors mediate rapid pattern separation in the hippocampal network. Science. 2007;317(5834):94-99.

27. Nakashiba $T$, et al. Young dentate granule cells mediate pattern separation, whereas old granule cells facilitate pattern completion. Cell. 2012;149(1):188-201.

28. Jessberger S, Zhao C, Toni N, Clemenson GD, Li Y, Gage FH. Seizure-associated, aberrant neurogenesis in adult rats characterized with retrovirus-mediated cell labeling. J Neurosci. 2007;27(35):9400-9407.

29. Wickersham IR, Finke S, Conzelmann KK, Callaway EM. Retrograde neuronal tracing with a deletion-mutant rabies virus. Nat Methods. 2007;4(1):47-49.

30. Etessami R, Conzelmann KK, Fadai-Ghotbi B, Natelson B, Tsiang H, Ceccaldi PE. Spread and pathogenic characteristics of a G-deficient rabies virus recombinant: an in vitro and in vivo study. J Gen Virol. 2000;81(Pt 9):2147-2153.

31. Bergami M, et al. A critical period for experience-dependent remodeling of adult-born neuron connectivity. Neuron. 2015;85(4):710-717.
32. Curia G, Longo D, Biagini G, Jones RS, Avoli M. The pilocarpine model of temporal lobe epilepsy. J Neurosci Methods. 2008;172(2):143-157.

33. Mathern GW, Adelson PD, Cahan LD, Leite JP. Hippocampal neuron damage in human epilepsy: Meyer's hypothesis revisited. Prog Brain Res. 2002;135:237-251.

34. Cavalheiro EA, Leite JP, Bortolotto ZA, Turski WA, Ikonomidou C, Turski L. Long-term effects of pilocarpine in rats: structural damage of the brain triggers kindling and spontaneous recurrent seizures. Epilepsia.1991;32(6):778-782.

35. Overstreet-Wadiche LS, Bromberg DA, Bensen AL, Westbrook GL. Seizures accelerate functional integration of adult-generated granule cells. J Neurosci. 2006;26(15):4095-4103.

36. McAuliffe JJ, et al. Altered patterning of dentate granule cell mossy fiber inputs onto CA3 pyramidal cells in limbic epilepsy. Hippocampus. 2011;21(1):93-107.

37. Hsu D. The dentate gyrus as a filter or gate: a look back and a look ahead. Prog Brain Res. 2007;163:601-613.

38. Heinemann U, Beck H, Dreier JP, Ficker E, Stabel J, Zhang CL. The dentate gyrus as a regulated gate for the propagation of epileptiform activity. Epilepsy Res Suppl. 1992;7:273-280.

39. Lothman EW, Stringer JL, Bertram EH. The dentate gyrus as a control point for seizures in the hippocampus and beyond. Epilepsy Res Suppl.
1992;7:301-313.

40. Krook-Magnuson E, Armstrong C, Bui A, Lew $\mathrm{S}$, Oijala M, Soltesz I. In vivo evaluation of the dentate gate theory in epilepsy. J Physiol (Lond). 2015;593(10):2379-2388.

41. Scharfman HE. The enigmatic mossy cell of the dentate gyrus. Nat Rev Neurosci. 2016;17(9):562-575.

42. Scharfman HE. The CA3 "backprojection" to the dentate gyrus. Prog Brain Res. 2007;163:627-637.

43. Shi Y, Ikrar T, Olivas ND, Xu X. Bidirectional global spontaneous network activity precedes the canonical unidirectional circuit organization in the developing hippocampus. JComp Neurol. 2014;522(9):2191-2208.

44. Yasuda M, Johnson-Venkatesh EM, Zhang H, Parent JM, Sutton MA, Umemori H. Multiple forms of activity-dependent competition refine hippocampal circuits in vivo. Neuron. 2011;70(6):1128-1142.

45. Singer BH, Gamelli AE, Fuller CL, Temme SJ, Parent JM, Murphy GG. Compensatory network changes in the dentate gyrus restore long-term potentiation following ablation of neurogenesis in young-adult mice. Proc Natl Acad Sci U S A. 2011;108(13):5437-5442.

46. Lagace DC, et al. Cdk5 is essential for adult hippocampal neurogenesis. Proc Natl Acad Sci U S A. 2008;105(47):18567-18571.

47. Wickersham IR, et al. Monosynaptic restriction of transsynaptic tracing from single, genetically targeted neurons. Neuron. 2007;53(5):639-647. 